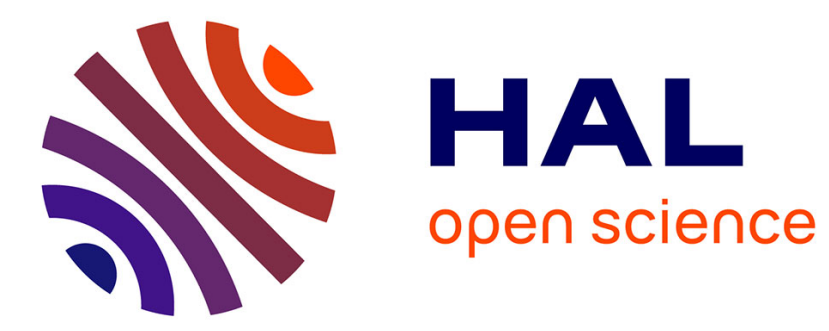

\title{
Anticipation and compensation for somatosensory deficits in object handling: evidence from a patient with large fibre sensory neuropathy
}

Ross Parry, Fabrice Sarlegna, Nathanael Jarrasse, Agnès Roby-Brami

\section{- To cite this version:}

Ross Parry, Fabrice Sarlegna, Nathanael Jarrasse, Agnès Roby-Brami. Anticipation and compensation for somatosensory deficits in object handling: evidence from a patient with large fibre sensory neuropathy. Journal of Neurophysiology, 2021, 10.1152/jn.00517.2020 . hal-03289253

\section{HAL Id: hal-03289253 \\ https://hal.science/hal-03289253}

Submitted on 24 Aug 2021

HAL is a multi-disciplinary open access archive for the deposit and dissemination of scientific research documents, whether they are published or not. The documents may come from teaching and research institutions in France or abroad, or from public or private research centers.
L'archive ouverte pluridisciplinaire HAL, est destinée au dépôt et à la diffusion de documents scientifiques de niveau recherche, publiés ou non, émanant des établissements d'enseignement et de recherche français ou étrangers, des laboratoires publics ou privés. 


\title{
Anticipation and compensation for somatosensory deficits in object handling: evidence from a patient with large fibre sensory neuropathy
}

3

4

\author{
Ross Parry ${ }^{1}$, Fabrice R. Sarlegna ${ }^{2}$, Nathanaël Jarrassé ${ }^{3}$, Agnès Roby-Brami ${ }^{3}$.
}

1. LINP2 - Laboratoire Interdisciplinaire en Neurosciences, Physiologie et Psychologie: Activité Physique, Santé et Apprentissages, UPL, Université Paris Nanterre, France.

2. Aix Marseille Univ, CNRS, ISM, Marseille, France

3. Institut des Systèmes Intelligents et de Robotique, Paris, France

10

11 Correspondence should be addressed to:

12 Ross Parry

13 Bâtiment Alice Miliat

14 UFR Sciences et Techniques des Activités Physiques et Sportives

15 Université Paris Nanterre

16200 avenue de la République

1792001 Nanterre Cedex 
The purpose of this study was to determine the contributions of feedforward and feedback processes on grip force regulation and object orientation during functional manipulation tasks.

One patient with massive somatosensory loss resulting from large fibre sensory neuropathy, and ten control participants were recruited. Three experiments were conducted: 1) perturbation to static holding; 2) discrete vertical movement; and 3) functional grasp and place. The availability of visual feedback was also manipulated to assess the nature of compensatory mechanisms. Results from experiment 1 indicated that both the deafferented patient and controls used anticipatory grip force adjustments prior to self-induced perturbation to static holding. The patient exhibited increased grip response time, but the magnitude of grip force adjustments remained correlated with perturbation forces in the self-induced and external perturbation conditions. In experiment 2, the patient applied peak grip force substantially in advance of maximum load force. Unlike controls, the patient's ability to regulate object orientation was impaired without visual feedback. In experiment 3, the duration of unloading, transport and release phases were longer for the patient, with increased deviation of object orientation at phase transitions. These findings show that the deafferented patient uses distinct modes of anticipatory control according to task constraints, and that responses to perturbations are mediated by alternative afferent information. The loss of somatosensory feedback thus appears to impair control of object orientation, while variation in the temporal organization of functional tasks may reflect strategies to mitigate object instability associated with changes in movement dynamics.

Key words: manual dexterity, somatosensory feedback, grip force control, sensory neuropathy 
43 New and noteworthy: This study evaluates the effects of sensory neuropathy on the scaling 44 and timing of grip force adjustments across different object handling tasks (i.e. holding, 45 vertical movement, grasping and placement). In particular, these results illustrate how novel 46 anticipatory and online control processes emerge in order to compensate for the loss of 47 somatosensory feedback. In addition, we provide new evidence on the role of somatosensory 48 feedback for regulating object orientation during functional prehensile movement. 
Grasping and using everyday objects implies the ability to anticipate and respond to dynamic task constraints (1), assuring regulation of forces and object orientation. Somatosensory feedback supports prehensile and manipulative function as cutaneous mechanoreceptors provide information on skin deformation while intrafusal muscle fibres, tendon organs and joint capsules transmit information regarding dynamic properties relevant to object mass and limb kinematics (2-4). Integration of these tactile and proprioceptive afferents assists to guide arm movement and coordinate finger forces applied to handheld objects. Experimental studies using dorsal rhizotomy procedures on non-human primates $(5,6)$, local anaesthetics/nerve blocks on healthy humans (7-13), or investigations on patients suffering from somatosensory deficits (14-16) demonstrate that tactile and proprioceptive dysfunction is associated with deficits in the accuracy and coordination of arm movements (17-19), and diminished manual dexterity (20-24).

Experimental studies of motor control in humans with somatosensory deficits have provided specific insights into the role of feedforward and feedback control mechanisms internal models allowing to anticipate the effects of movement kinematics upon inertial loads 
Of course, sensory feedback also is essential for reacting to external perturbations, in

that it informs the nervous system when generating an appropriate motor command with respect to changing task demands (33). For instance, when external forces are applied to a handheld object, somatosensory feedback enables a reflexive grip force increase $(34,35)$. This process is mediated by tactile afferents and long-latency stretch reflexes combined with fast volitional adjustments engaging transcortical pathways $(36,37)$. The ability to scale this "catch up" response to the magnitude of the perturbation is impaired when cutaneous feedback is compromised (34). Visual feedback has also been shown to contribute to the control of arm movements $(38,39)$ and dextrous manipulation $(40,41)$. But the extent to which this visual stream enables an individual to compensate for the loss of somatosensory afferents in object handling is less apparent (24). For instance, it remains to be determined how vision influences the ability to stabilize a handheld object through the course of movement, or respond to external perturbations in the absence of tactile or proprioceptive feedback.

Functional actions, such as grasping and moving an object, involve distinct phases characterized by changing task constraints (2). Feedforward control assures grip-load force coupling with variations in object acceleration, according to the specific constraints of each sub-goal of the task (42), while somatosensory signals continuously inform the nervous system of changes in mechanical events corresponding with phase transitions (i.e. object grasping to object unloading, unloading to lifting...) to ensure feedback control (2). Generally speaking, experiments on these types of naturalistic tasks are less common than those examining some functional primitive (i.e. reach, transport...) of a complex task $(43,44)$. Characterisation of a functional task which integrates grasp, transport and placement components may provide greater insight regarding the interplay of feedforward and feedback processes which underpin fine motor activities. In particular, further observation with 
deafferented patients may help to illustrate the role of somatosensory afferents in regulating grip forces and maintaining object orientation across the component phases of the functional movement.

In the present study, our objective was to examine the strategies used to stabilise grasp, adjust grip forces and maintain object orientation in the absence of somatosensory feedback. To this end, we compared the object handling abilities of one patient with large fibre sensory neuropathy and age-matched controls in a series of experiments with different task constraints. Perturbation to static holding, discrete vertical movement, and functional grasp and place paradigms were used in order to distinguish specific feedforward and feedback mechanisms. Visual feedback and the source of perturbation were also manipulated to better elucidate how the deafferented patient compensates for the loss of touch and proprioception. Based upon previous studies (26), we expected that the deafferented patient would use increased grip forces as a means to ensure object stability during perturbations to static holding and vertical movements. We also hypothesised that the deafferented patient's performance in perturbation to static holding and vertical movement would be compromised by removing visual feedback. Given the known interactions between feedforward and feedback control mechanisms in the functional grasp and place task (2), we further predicted that loss of somatosensory feedback would impair movement kinematics as well as the gripload force coupling and object orientation across the sequence of phases.

\section{MATERIALS AND METHODS}

\subsection{Participants}

Eleven participants were recruited: one deafferented patient (GL, a 67-year-old female) and ten adult controls ( 6 females/4 males, mean age $63 \pm 6$ years) with no known neurological or 
orthopaedic pathology affecting upper limb function. The deafferented patient suffered from a rare condition resulting in a massive yet specific, large-fibre sensory neuropathy (confirmed by sural nerve biopsy) (14). GL had thus lost somatosensory feedback from cutaneous receptors, muscle spindles and Golgi tendon organs (14). Consequently, this patient had chronic loss of proprioception and tactile sensation below the level of the nose.

All participants were tested for hand dominance using the Edinburg Handedness Inventory (45) prior to beginning the evaluation. Overall grip strength was evaluated using a hand dynamometer (LiteXpress $\mathrm{GmbH}$, Germany). In addition, GL responded to the QuickDASH questionnaire in order to measure self-perceived upper limb disability in daily life tasks (46). This study was approved by the ethics committee of Paris Descartes University and conducted in accordance with the declaration of Helsinki. All participants provided written consent prior to engaging in this experiment.

\subsection{Apparatus}

Object manipulation parameters were measured using an instrumented object (iBox), measuring $108 \times 70 \times 40 \mathrm{~mm}$ with a mass of $0.370 \mathrm{~kg}$ (see Figure 1A). The iBox is equipped with six load cells to measure the normal force applied to the six faces of the object, as well as an inertial measurement unit to record acceleration, rotational velocities and orientation (47).

Each load cell has a range of $0 \mathrm{~N}-50 \mathrm{~N}$ with a precision of $\pm 0.25 \mathrm{~N}$, measured by a set of 8 Bit analogue to digital converters (ADC). This device is calibrated on a flat surface to offset the mass of the iBox so that the base load cell measures $0 \mathrm{~N}$ when resting on a table and has a negative value when completely lifted from the table. Data was acquired at a sampling rate of $100 \mathrm{~Hz}$ and relayed to a personal computer via Bluetooth. All variables examined in this paper

were extracted from the raw data obtained during the three experiments. A $10 \mathrm{~Hz}$ low-pass 
147 Butterworth filter was used to remove low resolution artifacts for the purposes of illustration 148 in the present manuscript.

The experiment with GL was recorded in its entirety (with her signed consent) using a

digital camera. The first author (RP) was responsible for conducting each experiment.

Insert figure 1 about here

\subsection{Experiment 1: Perturbation to static holding}

2.3.1 Procedure. Participants were instructed to hold the iBox in a comfortable posture in front of their body, approximately $30 \mathrm{~cm}$ from the surface of the table with the elbow flexed and the shoulder in a neutral or slightly flexed position. From this position, load perturbations were introduced by striking the upper surface of the iBox by hand to produce a downward force pulse. Three conditions were evaluated: self-induced perturbation with visual feedback; external perturbation with visual feedback; and external perturbation without visual feedback (NB: previous tests indicated that self-induced perturbation without visual feedback was not feasible with the deafferented patient). For self-induced perturbation with visual feedback, participants used their free hand to strike the upper surface of the handheld iBox upon verbal command of the researcher. In the conditions involving an externally applied perturbation (with/without visual feedback), the researcher used their dominant hand to strike the iBox. For both the self-induced and external perturbation with vision conditions, participants were permitted to observe each action. A mask was applied over the participant's eyes for the external perturbation without visual feedback condition.

A demonstration for each condition was carried out prior to recording in order to illustrate the desired action when striking the iBox. A practice trial was used to ensure that instructions for each condition were understood. Force of the perturbation during each 
condition was varied in a semi-randomised manner according to the verbal instructions of the researcher ('stronger', 'softer'). Intervals between each perturbation event were staggered to prevent priming effects. Desired range of force perturbations was $1.5 \mathrm{~N}-16 \mathrm{~N}($ mean $=6 \mathrm{~N})$ with a force pulse duration of $\sim 70 \mathrm{~ms}$. Magnitude of force signals was inspected during recordings to ensure consistency in the strength of perturbations. Where required, certain trials were repeated to ensure comparability across participants for a total of ten trials. Each condition was conducted first with the non-dominant hand and then with the dominant hand.

2.3.2 Data extraction. Perturbation events were identified from the load cell on the upper surface of the iBox. Onset was defined as the data sample immediately preceding a threshold value of $0.25 \mathrm{~N}$. Grip force was measured as the mean grip forces recorded on the front and back load cells of the iBox. The following variables were then extracted for analysis:

- Baseline grip force during holding: minimum value for grip force during a $1 \mathrm{~s}$ time window prior to perturbation

- Grip response time: interval between perturbation onset and the time of maximum grip force

- Correlation for grip force scaling: Pearson correlation coefficient between the values of maximum grip force and maximum force applied to the upper load cell, across trials for a given condition

Ten perturbation trials for each condition on both dominant and non-dominant hands were selected for each participant. Trials for GL were selected to include a wide range of perturbation forces while trials with more variable acceleration during the static phase were eliminated. Trials in healthy participants were selected according to the maximum force on the upper load cell from the range of values observed with GL. In order to ensure the validity of comparisons, maximum force applied to the upper load cell, duration of the force pulse, 
and load rate parameters were examined (see S1.1-S1.3 for data visualization of stimulus parameters: https://figshare.com/s/840e0e8efb1e87e43157).

\section{https://doi.org/10.6084/m9.figshare.12888311}

No significant differences were found between GL and control subjects (results of statistical $\begin{array}{llll}\text { analysis } & \text { are } & \text { in } & \text { supplementary }\end{array}$ https://figshare.com/s/840e0e8efb1e87e43157)

https://doi.org/10.6084/m9.figshare.12888311

\subsection{Experiment 2: Vertical displacement of handheld object}

2.4.1 Procedure. In a seated position, participants held the iBox in a comfortable posture in front of their body as for experiment 1 . Participants were instructed to move the iBox on the vertical axis for a distance of approximately $30 \mathrm{~cm}$. Each trial consisted of one upward and one downward movement. A pause of approximately 1s was imposed between movements to ensure that each trial involved two discrete gestures (upward and downward). A verbal command was provided to signal the time of commencement for each direction. A ruler was used to demonstrate the appropriate movement amplitude prior to each trial. In the condition without visual feedback (blinded), a mask was placed over the participants' eyes. This mask was removed following each trial so that participants could adjust the orientation of the iBox as necessary. A total of 6 trials for the dominant and non-dominant hands were recorded for both directions under each condition (with and without visual feedback).

2.4.2 Data extraction. The instant of movement onset was detected using an interactive graphic interface allowing the selection of the inflection point in the acceleration signal. Individual movement trials were identified with windows beginning $500 \mathrm{~ms}$ prior to 
movement onset and lasting $2000 \mathrm{~ms}$. Grip force was measured as the mean grip forces recorded on the front and back load cells of the iBox (per experiment 1). Load force was measured as the product of the object's acceleration with the object's mass, using the method described in (48). Object orientation was calculated from the inertial motion unit data and expressed as the alpha angle, indicating the deviation of the longitudinal axis of the iBox from the vertical axis. The following variables were then extracted for analysis:

- Baseline grip force during holding: minimum value for grip force during the $500 \mathrm{~ms}$ prior to onset of movement

- Maximum acceleration: value for maximum acceleration during the movement window

- Maximum grip force: value for maximum grip force and the instant it occurred after movement onset

- Magnitude of grip force increase: difference between baseline and maximum grip force

- Time series correlation of grip force and load force: correlation coefficient for grip force and load force signals during the $1500 \mathrm{~ms}$ window from movement onset

- Ratio of maximum grip to maximum load: calculated by dividing the maximum grip force value from each trial by the maximum load force value from each trial

- Grip-load time delay: interval between the time points for the maximum grip force and the maximum load force values

- Average object angle: mean alpha angle calculated across time points for the duration of each trial

- Variability of object angle: standard deviation of the alpha angle calculated across time points for the duration of each trial 
Accelerometer data for GL could not be exploited for vertical movement without visual feedback due to large variations in iBox orientation during her movements.

\subsection{Experiment 3: Grasp, transport and placement of handheld object}

247

2.5.1 Procedure. Participants were in the seated position with their hand placed in the hand start area. The iBox was placed vertically before each participant, positioned in the parasagittal plane, $20 \mathrm{~cm}$ in front of the hand used. For each trial, participants were asked to grasp the object and place it on an $8 \mathrm{~cm}$ high platform located $15 \mathrm{~cm}$ distal to the initial position. This layout is illustrated in figure 1B. Three repetitions were carried out for the dominant and non-dominant hands.

2.5.2 Data extraction. Grip force and load force parameters were calculated using the same methods described in experiments 1 and 2. Transitions between unloading, lifting and placement phases were identified in an automated manner with reference to iBox load cell data, following the process described in $(43,49)$. Grasp onset was defined as the moment when the force applied to each of the front and back load cells exceeded $0.15 \mathrm{~N}$. Lift onset was the moment when the base load cell value was inferior to a threshold of $-3.4 \mathrm{~N}$ (based upon offset from object's mass). Placement was the moment when the base load cell returned to the threshold value of $-3.4 \mathrm{~N}$. Object release was defined as the moment when the mean of the forces applied to the two front and back load cells were inferior to $0.15 \mathrm{~N}$. Transport onset and transport end events were tagged manually from data in each trial using a graphic interface; these events corresponded with the end of the upward vertical acceleration and the beginning of the downward vertical acceleration respectively. From these events, five separate phases were identified: (1) unloading, between grasp onset and lifting onset; (2) lifting, between lift onset and transport onset; (3) transport, between transport onset and transport end; (4) 
descent, between transport end and placement; and (5) release phase, between placement and release.

Further to this, the occurrence of push and touch errors (43) were identified. Touch errors were defined as events where the sum of forces on the exposed (front, back, top and lateral) load cells exceeded $0.7 \mathrm{~N}$ before grasp onset or after release. A push error was detected as increased force $(>0.4 \mathrm{~N})$ on the base load cell during the unloading or release phases.

Based upon this, the following variables were then extracted for analysis:

- Durations for the unloading, lifting, transport, descent and release phases of the task

- Average grip-load ratio through unloading, lifting, transport, descent and release phases: a mean value calculated from the grip-load ratios from all time points within each respective phase

- Ratio of maximum grip to maximum load: calculated by dividing the maximum grip force value by the maximal load force value from each task

- Time series correlation of grip force and base load cell: correlation coefficient for grip force and base load sensor signals, calculated across the duration of unloading and release phases

- Grip-load time delay: interval between the time point for the maximum grip force and time point for the maximum load force

- Object angle at phase transitions: alpha angle at the grasp onset, lifting onset, transport onset, transport end, placement, and release end time points

- Average object angle through each respective phase: the mean alpha angle calculated across the time points for each of the unloading, lifting, transport, descent and release phases 
- Variability of object angle through each respective phase: the standard deviation calculated on the alpha angle across the time points within each respective phase of the task

- Occurrence of touch and push errors

\subsection{Statistical analysis}

Data was firstly analysed by examining each experimental task individually. Repeated measures analysis of variance (ANOVA) was performed on control data taking into account the different factors examined across the different experimental tasks (e.g. dominant / nondominant hand; visual feedback / absence of visual feedback; self-generated / external perturbation). These tests indicated that across control participants, there were no significant effects for the hand used in experiment 1 and experiment 2, and only 1 difference for variables examined in experiment 3 (grip-load time delay in the downward direction). Results for these statistical tests are provided in S2.2-S2.4: https://figshare.com/s/840e0e8efb1e87e43157). Due to the absence of significant differences between dominant and non-dominant hands, all data was pooled for subsequent statistical analysis with the deafferented patient.

Fisher transformations were performed prior to statistical comparison of correlation coefficients and Chi-squared tests were used for testing statistical difference in frequency of touch/push contact errors. Direct comparisons between values for GL and the controls data were carried out using the method described by (50), specifically designed for testing the mean of single subject data against a normative sample.

Secondly, further testing for common variables across different tasks (e.g. baseline grip force for vertical displacement and perturbation tasks) was performed upon the ensemble of the deafferented patient's data using a two-way ANOVA (hand used and experimental task as independent factors). Equivalent tests upon control group averages were conducted using 
repeated measures ANOVA with Greenhouse-Geisser adjustments where necessary. Statistical significance was set at $p=0.05$ and Bonferroni corrections were applied for multiple comparisons on post-hoc testing. All statistical analyses were conducted using Matlab, JASP and the SINGLIMS toolbox. Summary statistics in the results are expressed in terms of the mean and standard deviation (i.e. the ' \pm ' indicates intraindividual variability for G.L and interindividual variability for control subjects). Where boxplots are used to represent data, the central line corresponds to the median with the boxes indicating quartile values and whiskers the extremities.

The deafferented patient was evaluated as being right hand dominant (laterality quotient 80 ). The control group comprised 9 right handed participants (laterality quotient 90土8) and 1 left handed participant (laterality quotient -100 ). Maximum grip strength for GL was $17.2 \mathrm{~kg}$ for the dominant hand and $18.1 \mathrm{~kg}$ for the non-dominant hand. These values did not significantly differ to $29.1 \pm 6.5 \mathrm{~kg}$ for the dominant hand $(p=0.12)$ and $26.9 \pm 7.1 \mathrm{~kg}(p=0.27)$ for the nondominant hand in the control group (NB: control averages in the 6 females were $24.8 \pm 3.5 \mathrm{~kg}$ and $22.8 \pm 1.7 \mathrm{~kg}$ for dominant and non-dominant hands respectively). Differences between grip strength in the right and left hands of the control group were not statistically significant $(p=0.14)$. The deafferented patient scored $25 / 100$ on a QuickDASH questionnaire, indicating functional upper limb deficits with daily living tasks compared to females of a similar age group $(46,51)$.

\subsection{Experiment 1: Perturbation to static holding}

For the perturbation tasks, both the deafferented patient (GL) and the control group presented with increased grip forces in response to the iBox being struck. Figure 2 provides an example 
339 for each perturbation condition for GL and one representative control participant (C7).

340 Generally speaking, GL's baseline grip force appeared to fluctuate more than controls.

341 Similar to controls, GL was observed to have an increase in grip force prior to the

342 perturbation event in the self-induced condition. The timing of this grip force increase in the

343 self-induced condition, however, did appear to commence earlier for GL than for control

344 subjects (see figure 3).

Insert figures 2 and 3 about here

346

347

While GL appeared to have slightly greater baseline grip forces across perturbation conditions, these values were not statistically different from the control group (see figure 4A). Grip response time was significantly greater for GL than controls across the self-perturbation $(p=0.049)$, external perturbation with visual feedback $(p=0.003)$ and external perturbation without visual feedback ( $p=0.003$ ) conditions (see figure 4B). No significant difference was observed for grip force increase between GL and the control group in self perturbation $(4.7 \mathrm{~N} \pm 3.1 \mathrm{~N}$ vs $5.2 \mathrm{~N} \pm 3.0 \mathrm{~N} ; p=0.844)$ or external perturbation $(8.8 \mathrm{~N} \pm 5.3 \mathrm{~N}$ vs $6.1 \mathrm{~N} \pm 2.6 \mathrm{~N}$; $p=0.332$ ). However, average grip force increase for GL in the external perturbation without visual feedback condition was $7.9 \mathrm{~N}( \pm 5.6 \mathrm{~N})$, significantly greater than the control group at $4.3 \mathrm{~N}( \pm 3.39 \mathrm{~N} ; p=0.043)$.

Figure 4C provides a boxplot summary for the correlation of grip force scaling, which did not significantly differ between GL and the control group. Further detail on results from experiment 1 are provided S2.1—S2.2 (https://figshare.com/s/840e0e8efb1e87e43157).

Insert figure 4 about here

\subsection{Experiment 2: Vertical displacement of handheld object}

Qualitative differences were observed in the grip force dynamics of the deafferented patient and controls. Overall, GL presented with higher grip forces than controls. Figure 5 provides 
examples of an upward movement from the dominant hand for GL and one representative control participant (C5). While grip force variations appeared associated with variations in acceleration for controls, this was less evident for GL. In effect, relatively large grip forces were apparent even prior to movement initiation (see figure 5A). In the upward direction, GL's grip forces tended to decrease progressively during the duration of the movement (see figure 5A) but were more irregular in the downward direction (see examples of downward vertical movements in supplementary figure $\mathrm{S} 1.4$ and 1.5: https://figshare.com/s/840e0e8efb1e87e43157). In general, peak acceleration for GL (2.1 ms ${ }^{2} \pm 0.8 \mathrm{~ms}^{-2}$ for upward direction, $1.3 \mathrm{~ms}^{-2} \pm 0.4 \mathrm{~ms}^{-2}$ for downward direction) tended to be lower than controls $\left(4.0 \mathrm{~ms}^{-2} \pm 1.7 \mathrm{~ms}^{-2}\right.$ for upward direction, $2.9 \mathrm{~ms}^{-2} \pm 1.4 \mathrm{~ms}^{-2}$ for downward direction), although this was not statistically significant $(p=0.289, p=0.271)$.

\section{Insert figure 5 about here}

Figure 6 provides boxplot summaries for baseline grip force, maximum grip force and grip-load time delay during visually-guided vertical movements. In the upward direction, GL's baseline grip force was significantly higher than controls ( $p=0.007$; see figure 6A). Maximum grip force was greater for GL than controls ( $p=0.003$; see figure 6B). GL applied maximum grip force prior to maximum load force, significantly earlier than controls ( $p=0.004$; see grip-load time delay figure $6 \mathrm{C}$ ). The time series correlation between grip and load force for GL was $0.33( \pm .24)$, lower than controls at $0.57( \pm .25)$, but not statistically different after Fisher transformation $(p=0.35)$. Statistical analysis of the variability of the different variables for each participant (i.e. measures of intra-individual variability) revealed that standard deviation in the baseline grip force signals $(p<0.001)$, maximum grip force across trials $(p=0.001)$ and grip-load time delay across trials $(p<0.001)$ were greater for GL than for individuals of the control group. 
For visually-guided downward movements, no significant difference in baseline grip force was observed between GL and controls ( $p=0.061$; see figure 6A). The patient's maximum grip force however, was significantly greater than that of controls $(p=0.004$; see figure 6B). Time series correlation between grip and load force for GL was $-0.08( \pm 0.37)$ compared to $0.42( \pm 0.38)$ in controls, although this was not statistically significant $(p=0.241)$. Statistical analysis of the variability of the different variables for each participant (i.e. intra$(p=0.001)$, maximum grip force across trials $(p<0.001)$ and ratio of maximum grip to maximum load across trials $(p<0.001)$ were all greater for GL than the control group. Gripload time delay was abnormal and particularly variable for the deafferented patient. In effect, average grip-load time delay for GL was significantly earlier at $-621 \mathrm{~ms}( \pm 537 \mathrm{~ms})$ compared to $90 \mathrm{~ms}( \pm 125 \mathrm{~ms})$ in controls $(p<0.001)$ with variability between trials for GL $(537 \mathrm{~ms})$ being greater than for controls (range $21 \mathrm{~ms}-390 \mathrm{~ms} ; p=0.001$ ).

\section{Insert figure 6 about here}

Figure 7A shows that for vertical movement without visual feedback, GL exhibited irregular object orientation compared to controls (figure 7B). Mean object angle was significantly greater for GL in both upward and downward movement compared to controls $(p<0.001$; see figure $7 \mathrm{C})$. Object orientation also tended to be more variable for GL than controls during vertical movement without visual feedback. In the downward direction, variability of the continuous object angle signals for GL was $7.8^{\circ}\left( \pm 5.4^{\circ}\right)$, significantly greater than for individuals in the control group at $3.0^{\circ}\left( \pm 1.7^{\circ} ; p=.015\right)$. When visual feedback was available, no significant differences in object orientation were observed between GL and controls. Further statistical details on results from experiment 2 are provided in supplementary materials S2.3 (https://figshare.com/s/840e0e8efb1e87e43157). 


\subsection{Experimental task 3: Grasp, transport and placement of the object}

Grip force profiles for the deafferented patient appeared distinct from controls in the grasp, transport and place task. Controls exhibited maximum grip force during the lifting phase, corresponding with maximum vertical acceleration. GL tended to have a progressive increase in grip force through the transport and preparation for deposit phase. This contrast can be observed in figure 8. In addition, the duration of the transport phase appeared longer for GL. Variations in GL's object acceleration were observed late in the transport phase and followed by a strong mechanical impact at the base load sensor upon object placement (see figure $8 \mathrm{C}$ ). Touch errors involving force signal variations prior to grasp onset and following object release, as well as push errors involving variations in the base load sensor during unloading and release phases were more flagrant for GL than controls (see figure 8E).

\section{Insert figure 8 about here}

Durations of the component phases are indicated in the example trials provided in figure 8 while figure 9A provides a boxplot summary of phase durations in the grasp and place task. Overall, average duration for the functional grasp, transport and place task was $1.98 \mathrm{~s}( \pm 0.15 \mathrm{~s})$ for $\mathrm{GL}$, longer than for controls $(1.29 \pm 0.28 \mathrm{~s} ; p=0.017)$. More specifically, GL exhibited longer unloading $(p=0.044)$, transport $(p<0.001)$ and release phases $(p=0.038)$ than controls. Figure 9B shows that average grip-load force ratios were higher for GL through the unloading $(p=0.012)$ and descent $(p=0.012)$ phases compared to controls.

\section{Insert figure 9 about here}

Figure 9C shows that object angle was increased for GL at several phase transitions during the grasp and place task compared to controls. Statistically significant differences in object angle were seen at grasp onset ( $p<0.001$; c.f. touch errors below), lift onset $(p=0.019)$, 
transport end $(p=0.035)$ and placement $(p=0.033)$ time points. In addition to this, average object angle across the duration of the unloading phase was greater for GL than controls $\left(2.2^{\circ} \pm 1.3^{\circ}\right.$ v. $\left.0.8^{\circ} \pm 0.5^{\circ} ; p=0.029\right)$, and variability of alpha angle was greater during the release phase $\left(1.9^{\circ} \pm 0.9^{\circ}\right.$ v. $\left.0.6^{\circ} \pm 0.3^{\circ} ; p<0.001\right)$

A greater frequency of touch errors on grasp initiation $(p=0.022)$ and for push errors $(p=0.027)$ were recorded for GL than for controls (see figure 10A). Time series correlation of grip force and base load cell during the unloading phase was lower for GL than for controls $(p<0.001)$, while no significant difference was observed during the release phase (see figure 10B). Further statistical details on results from experiment 3 are provided in supplementary materials S2.4 (https://figshare.com/s/840e0e8efb1e87e43157).

$$
\text { Insert figure } 10 \text { about here }
$$

\subsection{Grip force control in the deafferented patient across experimental tasks}

Baseline grip force for GL was found to vary across all conditions of experiment 1 (perturbations of static holding) and experiment 2 (vertical upward and downward movements, with or without vision) combined $(p<0.001 ; \mathrm{F}=15.333 ; \mathrm{df}=6)$. Figure 11 provides a boxplot summary of the ensemble of GL's baseline grip force when holding the object across experiments 1 and 2. Baseline grip force during external perturbation without vision was greatest, with post-hoc testing indicating that it was greater than in all other conditions. In addition to this, baseline grip force was smallest in self-perturbation to static holding (with vision), with post-hoc testing indicating differences compared to external perturbation of static holding with vision $(p<0.001)$ and upward movements with vision $(p<0.001)$. No significant effect of hand was observed. 
For the deafferented patient, the ratio of maximum grip to maximum load force in vertical movements (experiment 2) with vision and the grasp and place task (experiment 3 ) were found to vary according to condition $(p=0.045 ; \mathrm{F}=3.529 ; \mathrm{df}=2)$. This ratio was greatest in downward movement and lowest in the grasp and place task, although post-hoc testing did not find any significant differences in pairwise comparisons. Comparison of grip-load time delay across vertical movements with vision and the grasp and place task yielded no significant differences. Further statistical details and tables for comparisons across experimental tasks are provided in supplementary materials S2.5 and 2.6 (https://figshare.com/s/840e0e8efb1e87e43157). https://doi.org/10.6084/m9.figshare.12888311

(1)

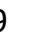

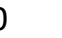

This study assessed the influence of somatosensory feedback on object manipulation by comparing the performance of a person with chronic large fibre sensory neuropathy to that of age-matched controls. As hypothesized, grip force analysis demonstrated that the deafferented patient (GL) generally used greater grip forces than the control group during object manipulation (vertical displacement, functional grasp and place). Moreover, GL had impaired temporal coupling of grip force adjustments with respect to voluntary movement dynamics (acceleration/load force) and perturbations to static holding (self-induced and externally induced). Without visual feedback, patient ability to maintain object orientation during discrete vertical movement was significantly impaired, which confirmed the second hypothesis of this study. This impairment appeared only partially compensated by visual feedback in the functional grasp and place task, as deviations in object angle were found to be superior to that of controls at several phase transitions. Increases in the duration and average grip-force ratios for certain phases further underscored kinematic and kinetic differences in the grasp and place task for the deafferented patient. 
487 effective movement execution, supporting and extending previous reports of impaired fine motor skills in deafferented individuals $(20,23,24,26)$. In addition, this study presents novel findings through comparison of motor performance across different task constraints. Firstly, grip force adjustments appeared earlier for (self-initiated) discrete movement and later for perturbations to static holding in the deafferented patient. Secondly, variations in the duration movements and the grasp and place task conveys the strengths and limits of the use of visual

\subsection{Feedforward modelling and pre-emptive regulation of grip force control}

The motor behaviour observed across the three experiments highlights different aspects of anticipatory control in the deafferented patient. In certain circumstances, GL demonstrated anticipatory finger force adjustments indicative of feedforward control processes. This was most evident in self-induced perturbation to static holding (experiment 1). Here the patient's grip force increased prior to impact, in preparation for the self-induced perturbation (figure 3). Also, in the functional grasp and place task, GL increased grip forces in parallel to the load variation during the unloading phase (figure $8 \mathrm{E}$ ), albeit with reduced sensitivity to load forces than controls (figures 9B, 10B). Increasing grip force in anticipation of load perturbation during holding $(35,52)$, and in parallel to load force increase during lifting $(9,43)$ is 
511 indicative of feedforward modelling where the consequences of one's intended action upon

512 the configuration of the body, object and environment are thought to be inferred based on an 513 efferent copy of the descending motor command (53).

The deafferented patient's ability for feedforward grip force control was less evident during discrete vertical movement (experiment 2). While grip forces of control participants tended to vary in parallel with object acceleration, the deafferented patient exhibited a more progressive change. For example, during upward vertical movements, GL’s grip forces tended to start high and decrease gradually (figure 5). GL's peak grip force occurred well in advance $(\sim 400 \mathrm{~ms})$ of peak acceleration (figure $6 \mathrm{C})$. The increased baseline was not only seen in upward movements (experiment 2, figure 6A) but also seemed somewhat apparent in the externally-induced perturbation conditions (experiment 1, figures 2,4A) and might be 522 interpreted as grip force adjustments in anticipation of the initiation of movement. In effect, 523 the deafferented patient seems to pre-emptively set high baseline grip forces for impending load variations.

We venture that specific task constraints influenced the mode of anticipatory control employed by the deafferented patient. In conditions when the hand was relatively still (self527 imposed perturbation, unloading phase of grasp and place), feedforward control for 528 synchronous grip-load force adjustment was a viable strategy for the patient (experiment 1, 529 figures 3, 4C; experiment 3 figure 10B). In tasks involving object displacement however (e.g. 530 vertical movement), the patient seemed to apply an increased grip force safety margin prior to 531 movement onset (experiment 2, figures 5A, 6C). In the case of external perturbation to 532 holding, healthy participants may exhibit preparatory actions ( $200 \mathrm{~ms}$ before event) (35). 533 Here again though, the deafferented patient still appeared to pre-set grip forces. Anticipatory 534 control for the deafferented patient may thus involve distinct cognitive strategies to 
compensate for situations where dynamic postural configuration or external loads are difficult to determine.

\subsection{Compensation for somatosensory deficits in reactive grip force scaling}

The experimental conditions examining externally induced perturbation of static holding, with vision and without vision, provide some insight into feedback mechanisms involved in reactive grip force scaling. In these conditions, the deafferented patient was found to have an increased grip response time when compared to the control group. Despite these temporal discrepancies, differences in the scaling of the grip force response with respect to the force applied to the handheld object were less pronounced than may have been expected (figure 4C). In the absence of tactile information pertaining to the state of the hand-object interface or proprioceptive stimulus pertaining to variations in joint position, the deafferented patient had limited direct access to mechanical features of the perturbation. From the observations presented here, it may thus be inferred that a deafferented patient uses other sensory modalities (albeit with greater latencies) to gauge the required grip force response.

For external perturbation with visual feedback, the deafferented patient may have adjusted grip forces based upon either her own arm movement or that of the person striking the object. Several studies $(39,54)$ have indicated that people are capable of identifying a change in their own arm trajectory and generate a corrective response within $\sim 150 \mathrm{~ms}$. Further, healthy adults prove highly receptive to the motion of other individuals (55). Using visual information of another person's body movements, adult participants can be highly accurate at determining the effects of that person's arm movement (56). Significant information may even be extrapolated from the other person's preparatory actions $(57,58)$. It is thus plausible 
that a deafferented patient can use visual perceptual capacities of human movement $(40,59$, 60) for determining the magnitude of the perturbation and thus the appropriate response.

The deafferented patient's ability to scale grip force during external perturbation in the blinded condition is more perplexing. Johansson et al (34) reported that certain participants managed to exhibit grip force adjustments in response to a pulling load despite having temporarily anaesthetised digits and their vision obscured. These participants may have relied on more proximal mechanoreceptors to mediate the grip force response. Similarly, Miall et al (60) found that deafferented patients detected unseen perturbations to reaching movements in a dynamically-perturbed environment (albeit with a higher threshold than controls). Several authors have also suggested that deafferented individuals may use vestibular feedback as a trigger for motion detection (61-63). In principle, the effects of arm oscillations upon the trunk and neck may provide the vestibular organs some crude information regarding load variations (25). In catching tasks, muscular cocontraction through the upper limb is commonly seen in anticipation of contact (64). It could be envisaged that in the case of static holding, an exaggerated pattern of cocontraction may assist in leveraging the effects of the distal perturbation and improve transmission of the mechanical stimulus to vestibular apparatus (59). The use of EMG and additional kinematic data would assist to verify this hypothesis.

The auditory stimulus associated with the impact represents another potential mode for the grip force adjustments observed during external perturbation without visual feedback. Using an experimental study on upper limb gestures, Danna \& Velay (65) illustrated that task sonification had a greater impact upon motor learning in deafferented patients (including GL) than controls. These authors advanced that these patients may indeed develop a supranormal capacity to process auditory information through cross-modal compensation as observed 
582 between sight and audition (66). Again, future studies manipulating auditory feedback with

583

584

585

586

587

588

589

590

591

592

593

594

595

596

597

598

599

600

601

602

603

604 deafferented individuals would be required to further examine this question.

\subsection{Object handling across sequential phases in functional grasp and place}

The differences in the duration of the phases of the grasp and place experiment provide insight into how the deafferented patient manages a functional object manipulation task. The unloading, transport and release phases were notably longer for the deafferented patient (figure 9A). Importantly, (vertical) object acceleration increased late in the transport phase and tended to spike upon placement (e.g. figure 8C, see also discussion of "micro-errors" in (43)). These changes in movement dynamics were associated with a corresponding grip force increase. Our findings thus suggest that the deafferented patient "restructures" the grasp and place task to overcome the somatosensory deficit. Increased grip force during unloading may ensure that grasp is indeed established prior to object displacement (10), while the greater duration in the transport phase may be associated with the use of a visuomotor control strategy (67). Vertical acceleration late in the transport phase may represent a kinematic strategy whereby a late vertical movement serves to ensure that the object arrives completely above the deposit platform. Finally, as upper limb proprioception is essential for controlling hand velocity (68), increased grip force during descent may serve to ensure object stability when considering the expected shock upon placement. Despite the reorganization in movement strategy, the deafferented patient was more irregular in the sequence of sub-goals as evidenced by a greater rate of "touch" and "push" errors. Changes in the grip force, acceleration profiles and temporal organisation of grasp and reach might thus be seen as both consequence of, and compensation for, the loss of tactile and proprioceptive feedback. 

handheld object represents one of the more novel findings of this study. Healthy participants are particularly skilled at maintaining vertical object orientation across tasks (43). In contrast, the deafferented patient exhibited marked difficulties to stabilize the object. This was most apparent at moments which coincided with changes in task dynamics in the functional prehensile task, those being grasp onset, lift onset, transport end and object placement. Again,

611 it is likely that deafferented patients' impairments in intersegmental coordination (68) at these 612 instants make phase transitions particularly vulnerable to instability of the handheld object.

613 Given the deafferented patient's impaired ability to maintain object orientation during vertical 614 movement in the absence of visual feedback (experiment 2), it appears that peripheral 615 somatosensory feedback is critical for maintaining object orientation as the task constraints 616 change with each sub-goal. Recent experimental findings (69) have suggested that 617 proprioception is necessary for body schema plasticity which underlies tool use. As a 618 consequence, a deafferented patient may tend to use more external reference frames than 619 healthy participants, which is consistent with what (70) reported for GL in a perceptual task. 620 However, in an object manipulation task, as in the present study, visual feedback control of 621 the hand-object system with respect to the external environment likely requires greater 622 processing time than proprioceptive feedback control (71), and may thus be more vulnerable 623 to difficulty with maintaining the vertical orientation of the handheld object at dynamic phase 624 transitions. Furthermore, GL was recently found to have altered patterns of finger enslaving 625 (resulting in greater individuation) (23), and this may render the task of managing the torque generated between thumb and fingers, more complex than for a healthy participant.

\section{$627 \quad 4.4$ Limitations of the study}

628 Several limitations should be recognized in the current study. Most importantly, perturbation 629 stimulus from experiment 1 was not precisely controlled via an automated process.

630 Nonetheless, the methods used here sought to limit the consequences through selection of 
comparable stimulus based upon the magnitude, duration and loading rate of the force pulses (per https://figshare.com/s/840e0e8efb1e87e43157). https://doi.org/10.6084/m9.figshare.12888311

In addition to this, further experimental apparatus including EMG and kinematic analysis would have provided further insight into the coordination of muscular activity and movement of the upper limb more generally. Finally, the present study included a modest number of trials for each experimental condition and only one deafferented patient. The results observed with GL in the current study may not necessarily generalize to other deafferented patients. In effect, differences in dexterous motor control was observed across deafferented patients in prior studies $(25,60)$. Accordingly, we would anticipate that other individuals may potentially employ alternative perceptual strategies for informing fast volitional responses to perturbations, or motor planning strategies in restructuring functional prehensile tasks.

\section{CONCLUSION}

A patient with chronic large fibre sensory neuropathy used different motor control strategies with respect to controls, highlighting the role of somatosensory feedback for object manipulation. Feedforward strategies were used for anticipatory grip force adjustment during self-induced perturbation and the unloading phase of a grasp and lift task in the deafferented and control participants, although increased grip-load ratios and increased response times illustrated that the integrity of this system was less effective when deprived of somatosensory feedback (26). Also, pre-emptive changes in baseline grip forces suggested that the deafferented patient used a cognitive strategy to estimate impending task dynamics and set grip force safety margins accordingly.

Despite temporal delays, the correlation of reactive grip force scaling suggests that alternative afferent signals (vision, not excluding audition and vestibular system) partially 
657 compensated for the loss of tactile and proprioceptive signals. Recognising an individual 658 aptitude with remaining sensory modalities may explain differences in motor performance 659 between deafferented patients (25), or be harnessed using assistive devices involving sensory 660 substitution (72). In a functional grasp and place task, certain phases were found to be 661 prolonged in the deafferented patient, while other phases were characterized by increased 662 grip-load force ratios. In addition, orientation of the object angle at several phase transitions 663 deviated more greatly for the deafferented patient than for control participants. This illustrates 664 the functional consequences of peripheral lesions to the somatosensory system and underscores the use of novel strategies used by the deafferented patient to mitigate deficits in everyday tasks such as object handling.

Acknowledgments: We would like to acknowledge the important contributions of the 669 reviewers and the associate editor during the revision of the present manuscript. We also wish to thank those who gave their time to participate in this study. In particular, we are very

671 grateful to the patient, GL, who sadly passed away (due to an unrelated medical condition) 672 during revision of this article. We extend our heartfelt condolences to those who knew her, 673 and dedicate this paper to her memory.

674 Funding: This work was supported by funding from the Labex SMART project (ANR-11675 LABX-65), Investissements d'Avenir programme (ANR-11-DEX-0004-02) as well as the 676 CNRS AUTON initiative (project SUBLIMA).

\section{References}


2. Johansson RS, Flanagan JR. Coding and use of tactile signals from the fingertips in object manipulation tasks. Nature Reviews Neuroscience 10: 345-359, 2009. doi: 10.1038/nrn2621.

3. Proske U, Gandevia SC. The Proprioceptive Senses: Their Roles in Signaling Body Shape, Body Position and Movement, and Muscle Force. Physiological Reviews 92: 1651-1697, 2012. doi: 10.1152/physrev.00048.2011.

4. Pruszynski JA, Flanagan JR, Johansson RS. Fast and accurate edge orientation processing during object manipulation. eLife 7: e31200, 2018. doi: 10.7554/eLife.31200.

5. Taub E, Goldberg IA, Taub P. Deafferentation in monkeys: Pointing at a target without visual feedback. Experimental Neurology 46: 178-186, 1975. doi: 10.1016/0014-4886(75)90040-0.

6. Gauthier GM, Mussa Ivaldi F. Oculo-manual tracking of visual targets in monkey: role of the arm afferent information in the control of arm and eye movements. Experimental Brain Research 73: 138-154, 1988. doi: 10.1007/BF00279668.

7. Augurelle A-S, Smith AM, Lejeune T, Thonnard J-L. Importance of cutaneous feedback in maintaining a secure grip during manipulation of hand-held objects. J Neurophysiol 89: 665671, 2003. doi: 10.1152/jn.00249.2002.

8. Carteron A, McPartlan K, Gioeli C, Reid E, Turturro M, Hahn B, Benson C, Zhang W. Temporary Nerve Block at Selected Digits Revealed Hand Motor Deficits in Grasping Tasks. Front Hum Neurosci 10, 2016. doi: 10.3389/fnhum.2016.00596.

9. Johansson RS, Westling G. Roles of glabrous skin receptors and sensorimotor memory in automatic control of precision grip when lifting rougher or more slippery objects. Exp Brain Res 56: 550-564, 1984.

10. Monzée J, Lamarre Y, Smith AM. The effects of digital anesthesia on force control using a precision grip. J Neurophysiol 89: 672-683, 2003. doi: 10.1152/jn.00434.2001.

11. Nowak DA, Hermsdörfer J, Glasauer S, Philipp J, Meyer L, Mai N. The effects of digital anaesthesia on predictive grip force adjustments during vertical movements of a grasped object: Digital anaesthesia and predictive grip force adjustments. European Journal of Neuroscience 14: 756-762, 2001. doi: 10.1046/j.0953-816x.2001.01697.x.

12. Westling G, Johansson RS. Factors influencing the force control during precision grip. Exp Brain Res 53: 277-284, 1984. doi: 10.1007/BF00238156.

13. Witney AG, Wing A, Thonnard J-L, Smith AM. The cutaneous contribution to adaptive precision grip. Trends Neurosci 27: 637-643, 2004. doi: 10.1016/j.tins.2004.08.006.

14. Forget R, Lamarre Y. Rapid elbow flexion in the absence of proprioceptive and cutaneous feedback. Hum Neurobiol 6: 27-37, 1987.

15. Medina J, Jax SA, Brown MJ, Coslett HB. Contributions of Efference Copy to Limb Localization: Evidence from Deafferentation. Brain Res 1355: 104-111, 2010. doi: 10.1016/j.brainres.2010.07.063.

16. Nowak DA, Hermsdörfer J. Selective deficits of grip force control during object manipulation in patients with reduced sensibility of the grasping digits. Neuroscience Research 47: 65-72, 2003. doi: 10.1016/S0168-0102(03)00182-2. 
17. Jayasinghe SAL, Sarlegna FR, Scheidt RA, Sainburg RL. The neural foundations of handedness: insights from a rare case of deafferentation. Journal of Neurophysiology 124: 259 267, 2020. doi: 10.1152/jn.00150.2020.

18. Polit A, Bizzi E. Characteristics of motor programs underlying arm movements in monkeys. $J$ Neurophysiol 42: 183-194, 1979. doi: 10.1152/jn.1979.42.1.183.

19. Sarlegna FR, Sainburg RL. The Roles of Vision and Proprioception in the Planning of Reaching Movements. Adv Exp Med Biol 629: 317-335, 2009. doi: 10.1007/978-0-387-77064$2 \_16$.

20. Rothwell JC, Traub MM, Day BL, Obeso JA, Thomas PK, Marsden CD. Manual motor performance in a deafferented man. Brain 105: 515-542, 1982. doi: 10.1093/brain/105.3.515.

21. Nowak DA, Hermsdörfer J, Marquardt C, Topka H. Moving objects with clumsy fingers: how predictive is grip force control in patients with impaired manual sensibility? Clin Neurophysiol 114: 472-487, 2003.

22. Melchior H, Vatine J-J, Weiss PPL. Is there a relationship between light touch-pressure sensation and functional hand ability? Disability and Rehabilitation 29: 567-575, 2007. doi: $10.1080 / 09638280600902547$.

23. Cuadra C, Falaki A, Sainburg R, Sarlegna FR, Latash ML. Case Studies in Neuroscience: The central and somatosensory contributions to finger interdependence and coordination: lessons from a study of a "deafferented person". J Neurophysiol 121: 2083-2087, 2019. doi: 10.1152/jn.00153.2019.

24. Miall RC, Rosenthal O, Ørstavik K, Cole JD, Sarlegna FR. Loss of haptic feedback impairs control of hand posture: a study in chronically deafferented individuals when grasping and lifting objects. .

25. Hermsdörfer J, Elias Z, Cole JD, Quaney BM, Nowak DA. Preserved and Impaired Aspects of Feed-Forward Grip Force Control After Chronic Somatosensory Deafferentation. Neurorehabilitation and Neural Repair 22: 374-384, 2008. doi: 10.1177/1545968307311103.

26. Nowak DA, Glasauer S, Hermsdörfer J. How predictive is grip force control in the complete absence of somatosensory feedback? Brain 127: 182-192, 2004. doi: 10.1093/brain/awh016.

27. Nowak DA, Hermsdörfer J. Objective evaluation of manual performance deficits in neurological movement disorders. Brain Research Reviews 51: 108-124, 2006. doi: 10.1016/j.brainresrev.2005.10.003.

28. Thonnard J-L, Detrembleur C, Van den Bergh PYK. Assessment of hand function in a patient with chronic sensory demyelinating neuropathy. Neurology 49: 253-257, 1997. doi: 10.1212/WNL.49.1.253.

29. Flanagan JR, Tresilian JR. Grip-load force coupling: a general control strategy for transporting objects. J Exp Psychol Hum Percept Perform 20: 944-957, 1994.

30. Flanagan JR, Bowman MC, Johansson RS. Control strategies in object manipulation tasks. Current Opinion in Neurobiology 16: 650-659, 2006. doi: 10.1016/j.conb.2006.10.005.

31. Nowak DA, Glasauer S, Hermsdörfer J. Force control in object manipulation-A model for the study of sensorimotor control strategies. Neuroscience \& Biobehavioral Reviews 37: 15781586, 2013. doi: 10.1016/j.neubiorev.2013.06.003. 
32. Hermsdörfer J, Hagl E, Nowak DA. Deficits of anticipatory grip force control after damage to peripheral and central sensorimotor systems. Human Movement Science 23: 643-662, 2004. doi: 10.1016/j.humov.2004.10.005.

33. Scott SH, Cluff T, Lowrey CR, Takei T. Feedback control during voluntary motor actions. Current Opinion in Neurobiology 33: 85-94, 2015. doi: 10.1016/j.conb.2015.03.006.

34. Johansson RS, Riso R, Häger C, Bäckström L. Somatosensory control of precision grip during unpredictable pulling loads. I. Changes in load force amplitude. Exp Brain Res 89: 181191, 1992.

35. Johansson RS, Westling G. Programmed and triggered actions to rapid load changes during precision grip. Experimental Brain Research 71, 1988. doi: 10.1007/BF00247523.

36. Macefield VaughanG, Rothwell JohnC, Day BrianL. The contribution of transcortical pathways to long-latency stretch and tactile reflexes in human hand muscles. Exp Brain Res 108, 1996. doi: 10.1007/BF00242912.

37. Macefield VG, Johansson RS. Loads applied tangential to a fingertip during an object restraint task can trigger short-latency as well as long-latency EMG responses in hand muscles. Experimental Brain Research 152: 143-149, 2003. doi: 10.1007/s00221-003-1421-9.

38. Ghez C, Gordon J, Ghilardi MF. Impairments of reaching movements in patients without proprioception. II. Effects of visual information on accuracy. J Neurophysiol 73: 361-372, 1995. doi: $10.1152 /$ jn.1995.73.1.361.

39. Saunders JA, Knill DC. Humans use continuous visual feedback from the hand to control fast reaching movements. Exp Brain Res 152: 341-352, 2003. doi: 10.1007/s00221-003-1525-2.

40. Sarlegna FR, Malfait N, Bringoux L, Bourdin C, Vercher J-L. Force-field adaptation without proprioception: Can vision be used to model limb dynamics? Neuropsychologia 48: 60-67, 2010. doi: 10.1016/j.neuropsychologia.2009.08.011.

41. Takamuku S, Gomi H. Better grip force control by attending to the controlled object: Evidence for direct force estimation from visual motion. Scientific Reports 9: 13114, 2019. doi: $10.1038 / \mathrm{s} 41598-019-49359-8$.

42. Danion F, Sarlegna FR. Can the Human Brain Predict the Consequences of Arm Movement Corrections When Transporting an Object? Hints from Grip Force Adjustments. J Neurosci 27: 12839-12843, 2007. doi: 10.1523/JNEUROSCI.3110-07.2007.

43. Martin-Brevet S, Jarrassé N, Burdet E, Roby-Brami A. Taxonomy based analysis of force exchanges during object grasping and manipulation. PLOS ONE 12: e0178185, 2017. doi: 10.1371/journal.pone.0178185.

44. Schambra HM, Parnandi A, Pandit NG, Uddin J, Wirtanen A, Nilsen DM. A Taxonomy of Functional Upper Extremity Motion. Frontiers in Neurology 10, 2019. doi: 10.3389/fneur.2019.00857.

45. Oldfield RC. The assessment and analysis of handedness: the Edinburgh inventory. Neuropsychologia 9: 97-113, 1971.

46. Hunsaker FG, Cioffi DA, Amadio PC, Wright JG, Caughlin B. The American academy of orthopaedic surgeons outcomes instruments: normative values from the general population. $J$ Bone Joint Surg Am 84: 208-215, 2002. doi: 10.2106/00004623-200202000-00007. 
47. Jarrassé N, Kuhne M, Roach N, Hussain A, Balasubramanian S, Burdet E, Roby-Brami A. Analysis of grasping strategies and function in hemiparetic patients using an instrumented object. In: Rehabilitation Robotics (ICORR), 2013 IEEE International Conference on. IEEE, 2013, p. 1-8.

48. Nowak DA, Hermsdörfer J, Topka H. When Motor Execution Is Selectively Impaired: Control of Manipulative Finger Forces in Amyotrophic Lateral Sclerosis. Motor Control 7: 304-320, 2003. doi: 10.1123/mcj.7.3.304.

49. Parry R, Macias Soria S, Pradat-Diehl P, Marchand-Pauvert V, Jarrassé N, Roby-Brami A. Effects of Hand Configuration on the Grasping, Holding, and Placement of an Instrumented Object in Patients With Hemiparesis. Front Neurol 10, 2019. doi: 10.3389/fneur.2019.00240.

50. Crawford JR, Garthwaite PH. Investigation of the single case in neuropsychology: confidence limits on the abnormality of test scores and test score differences. Neuropsychologia 40: 11961208, 2002. doi: 10.1016/S0028-3932(01)00224-X.

51. Angst F, Schwyzer H-K, Aeschlimann A, Simmen BR, Goldhahn J. Measures of adult shoulder function: Disabilities of the Arm, Shoulder, and Hand Questionnaire (DASH) and Its Short Version (QuickDASH), Shoulder Pain and Disability Index (SPADI), American Shoulder and Elbow Surgeons (ASES) Society Standardized Shoulder Assessment Form, Constant (Murley) Score (CS), Simple Shoulder Test (SST), Oxford Shoulder Score (OSS), Shoulder Disability Questionnaire (SDQ), and Western Ontario Shoulder Instability Index (WOSI). Arthritis Care \& Research 63: S174-S188, 2011. doi: 10.1002/acr.20630.

52. Nowak DA, Hermsdörfer J, Rost K, Timmann D, Topka H. Predictive and reactive finger force control during catching in cerebellar degeneration. The Cerebellum 3: 227-235, 2004. doi: $10.1080 / 14734220410019057$.

53. Wolpert DM, Miall RC, Kawato M. Internal models in the cerebellum. Trends Cogn Sci (Regul Ed) 2: 338-347, 1998.

54. Sarlegna FR, Blouin J, Vercher J-L, Bresciani J-P, Bourdin C, Gauthier GabrielM. Online control of the direction of rapid reaching movements. Experimental Brain Research 157, 2004. doi: 10.1007/s00221-004-1860-y.

55. Blake R, Shiffrar M. Perception of Human Motion. Annual Review of Psychology 58: 47-73, 2007. doi: 10.1146/annurev.psych.57.102904.190152.

56. Knoblich G, Flach R. Predicting the Effects of Actions: Interactions of Perception and Action. Psychological Science 12: 467-472, 2001. doi: 10.1111/1467-9280.00387.

57. Auvray M, Hoellinger T, Hanneton S, Roby-Brami A. Perceptual Weight Judgments When Viewing One's Own and others' Movements under Minimalist Conditions of Visual Presentation. Perception 40: 1081-1103, 2011. doi: 10.1068/p6879.

58. Vaziri-Pashkam M, Cormiea S, Nakayama K. Predicting actions from subtle preparatory movements. Cognition 168: 65-75, 2017. doi: 10.1016/j.cognition.2017.06.014.

59. Fleury M, Bard C, Teasdale N, Paillard J, Cole J, Lajoie Y, Lamarre Y. Weight judgment: The discrimination capacity of a deafferented subject. Brain 118: 1149-1156, 1995. doi: 10.1093/brain/118.5.1149.

60. Miall RC, Kitchen NM, Nam S-H, Lefumat H, Renault AG, Ørstavik K, Cole JD, Sarlegna FR. Proprioceptive loss and the perception, control and learning of arm movements in humans: 
evidence from sensory neuronopathy. Experimental Brain Research 236: 2137-2155, 2018. doi: $10.1007 / \mathrm{s} 00221-018-5289-0$.

61. Bringoux L, Scotto Di Cesare C, Borel L, Macaluso T, Sarlegna FR. Do Visual and Vestibular Inputs Compensate for Somatosensory Loss in the Perception of Spatial Orientation? Insights from a Deafferented Patient. Front Hum Neurosci 10, 2016. doi: 10.3389/fnhum.2016.00181.

62. Cole JD, Sedgwick EM. The perceptions of force and of movement in a man without large myelinated sensory afferents below the neck. J Physiol 449: 503-515, 1992.

63. Miall RC, Ingram HA, Cole JD, Gauthier GM. Weight estimation in a "deafferented" man and in control subjects: are judgements influenced by peripheral or central signals? Exp Brain Res 133: 491-500, 2000.

64. Lacquaniti F, Maioli C. Anticipatory and reflex coactivation of antagonist muscles in catching. Brain Research 406: 373-378, 1987. doi: 10.1016/0006-8993(87)90810-9.

65. Danna J, Velay J-L. On the Auditory-Proprioception Substitution Hypothesis: Movement Sonification in Two Deafferented Subjects Learning to Write New Characters. Front Neurosci 11, 2017. doi: 10.3389/fnins.2017.00137.

66. Lessard N, Paré M, Lepore F, Lassonde M. Early-blind human subjects localize sound sources better than sighted subjects. Nature 395: 278-280, 1998. doi: 10.1038/26228.

67. Bagesteiro LB, Sarlegna FR, Sainburg RL. Differential influence of vision and proprioception on control of movement distance. Exp Brain Res 171: 358, 2005. doi: 10.1007/s00221-005-0272y.

68. Sainburg RL, Ghilardi MF, Poizner H, Ghez C. Control of limb dynamics in normal subjects and patients without proprioception. Journal of Neurophysiology 73: 820-835, 1995. doi: 10.1152/jn.1995.73.2.820.

69. Cardinali L, Brozzoli C, Luauté J, Roy AC, Farnè A. Proprioception Is Necessary for Body Schema Plasticity: Evidence from a Deafferented Patient. Front Hum Neurosci 10, 2016. doi: $10.3389 /$ fnhum.2016.00272.

70. Arnold G, Sarlegna FR, Fernandez LG, Auvray M. Somatosensory Loss Influences the Adoption of Self-Centered Versus Decentered Perspectives. Frontiers in Psychology 10, 2019. doi: 10.3389/fpsyg.2019.00419.

71. Cameron BD, de la Malla C, López-Moliner J. The role of differential delays in integrating transient visual and proprioceptive information. Front Psychol 5, 2014. doi: 10.3389/fpsyg.2014.00050.

72. Kristjánsson Á, Moldoveanu A, Jóhannesson ÓI, Balan O, Spagnol S, Valgeirsdóttir VV, Unnthorsson R. Designing sensory-substitution devices: Principles, pitfalls and potential1. Restorative Neurology and Neuroscience 34: 769-787, 2016. doi: 10.3233/RNN-160647. 
880

881

882

883

884

885

886

887

888

889

890

891

892

893

894

895

896

897

898

899

900

901

902

903

904

905

906

907

908

909

910

911

Figure 1. Illustration of the iBox device and the experimental setup. (A) The iBox instrumented object. (B) Layout of the tabletop for the grasp, transport and place task. Initial positions of the iBox and hand start area are indicated by the dotted lines. The gray shaded rectangle indicates the deposit platform.

Figure 2. Experiment1: Representative examples of grip force profiles for perturbation to static holding for the deafferented patient (GL, red) and one control subject (C7, blue). The perturbation is indicated by the signal from the upper load cell, in black. (A/B) Grip force during self-induced perturbation. (C/D) Grip force during external perturbation with visual feedback. (E/F) Grip force during external perturbation without visual feedback. Vertical lines indicate the moment of the strike to the upper surface of the iBox. Examples have been specifically chosen with comparable force perturbation $(9 \mathrm{~N} \pm 0.7 \mathrm{~N})$.

Figure 3. Experiment 1: Preparatory grip force increase in self-induced perturbation. Grip force profiles for the deafferented patient (GL, red, left panel) and one representative control subject (C7, blue, right panel) during the 500ms preceding the strike to the upper surface of the iBox. Vertical line at $0 \mathrm{~ms}$ indicates the moment of strike to the upper surface of the iBox. Arrows indicate approximate onset of grip force increase.

Figure 4. Experiment 1: Boxplot summary of results for perturbation of static holding in selfinduced (Self), externally induced with visual feedback (External), and externally induced without visual feedback (Ext-Blinded) conditions. (A) Baseline grip force prior to perturbation. (B) Grip response time between peak force upon the upper surface of iBox and maximal grip force. (C) Pearson correlation between maximum force of strike to upper load cell and the maximum grip forces (calculated on $n=20$ trials per subject). Blue boxplot data indicates mean values for the control group, red lines indicate mean values for the deafferented patient. Blue asterisks indicate significant results for post-hoc tests between conditions in the control group. Red asterisks indicate significant differences between the deafferented patient and the control group using the method described by (50). * $=p<0.05, * *$ $=p<0.01 * * *=p<0.001$.

Figure 5. Experiment 2: Examples of upward vertical movements with the iBox for the deafferented patient (GL, red) and one representative control subject (C5, blue). (A/B) Grip force profiles. (C/D) Vertical (along the Y axis) acceleration of the object. (E/F) Alpha angle of the object, indicating deviation from the vertical. Vertical lines indicate onset of vertical movement. 
912 Figure 6. Experiment 2: Boxplot summary of results for upward vertical movements. (A)

913 Baseline grip force in visually guided movements. (B) Maximum grip force in visually guided 914 movements. (C) Grip-load time delay for visually guided movements. Blue boxplot data 915 indicates values for control group data, red lines indicate mean values for deafferented patient. 916 Red asterisks indicate significant differences between the deafferented patient and the control 917 group using the method described in (50). ** $=p<0.01, * * *=\mathrm{p}<0.001$.

918 Figure 7. Experiment 2: Object orientation during vertical movement. (A/B) Object 919 orientation profiles during upward movement without visual feedback for the deafferented 920 patient (GL, red) and one representative control subject $(\mathrm{C} 1$, blue). (C) Boxplot summary of 921 average object orientation during vertical movement. Blue boxplot data indicates values for 922 control group data, red lines indicate mean values for deafferented patient. Red asterisks 923 indicate significant differences between the deafferented patient and the control group using 924 the method described in (50).*** $=\mathrm{p}<0.001$. Lightning symbol indicates a break in the 925 vertical axis for figure $7 \mathrm{C}$.

926 Figure 8. Experiment 3: Examples of signals from grasp, transport and place task for 927 deafferented patient (GL, red) and one control subject (C1, blue). (A/B) Angle measuring the deviation of the iBox from the vertical. (C/D) Vertical (along the Y axis) acceleration of the iBox. (E/F) Grip force (plain line) during grasp, transport and place task. Object loading (dashed lines) is indicated using force data from load cell on the base of the iBox. Vertical lines indicate the times of transitions between phases $g r=$ grasp onset; $l i=$ lift onset, $t r=$ transport onset; $t e=$ transport end; $p l=$ placement; $r e=$ release end. Time $=0$ at $t g$. Arrows indicate instance of touch errors prior to establishing grasp and push errors during the 934 unloading and release phases. Figure 9. Boxplot summary of results for grasp, transport and place task. (A) Duration of component phases during the task. (B) Average grip-load force ratio during the different phases. (C) Object angle at transitions between phases of the task. Blue boxplot data indicates values for control group data (crosses indicate outliers), red lines indicate mean values for deafferented patient. Red asterisks indicate significant results for comparisons between the

940 deafferented patient and the control group using the method described by $(50) . *=p<0.05, * *$ $941=p<0.01, * * *=\mathrm{p}<0.001$. 
943 Figure 10. (A) Frequency of touch errors prior to onset of grasp, and push errors through the 944 unloading phase. NB: Absence of variation precluded establishing quartile values for touch 945 error boxplot, for this reason, individual values are projected on the median bar line for 946 clarity. Crosses indicate outliers. * $=$ Chi-squared test $p<0.05$. (B) Correlation between grip 947 force and force of the base load sensor of instrumented object during unloading and release 948 (loading) phase. Correlation data is presented as an absolute value. Deafferented patient data 949 is indicated in red while control subject data is indicated in blue. Statistical differences for 950 correlation values have been calculated after Fisher transformation. $* * *=p<0.001$ using 951 method described in (50).

952 Figure 11. Baseline grip force for the deafferented patient across tasks with static holding 953 phases. Black asterisks indicate results from post-hoc testing. $*=p<0.05, * *=p<0.01, * * *=$ $954 \mathrm{p}<0.001$. 


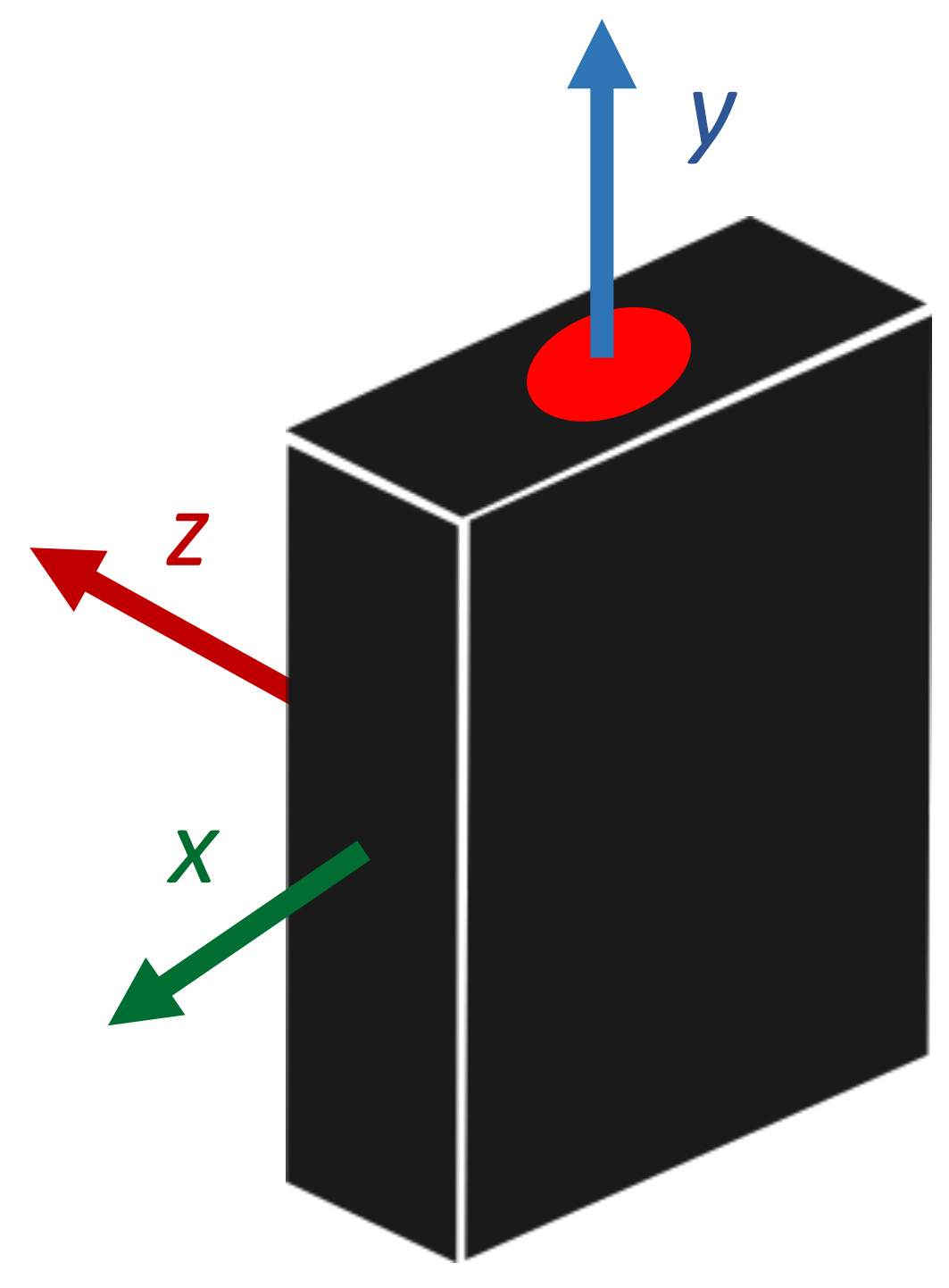

A

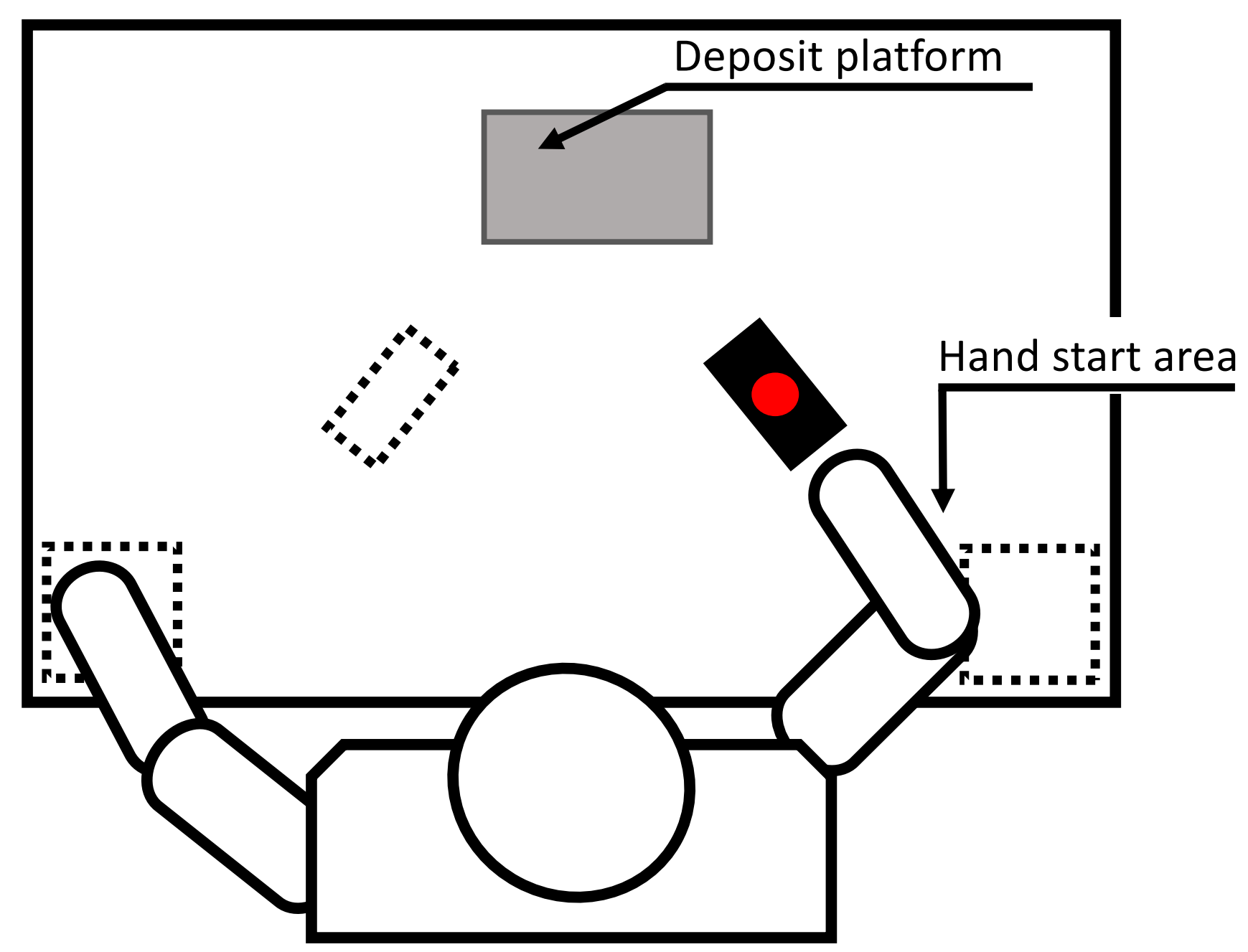

B 

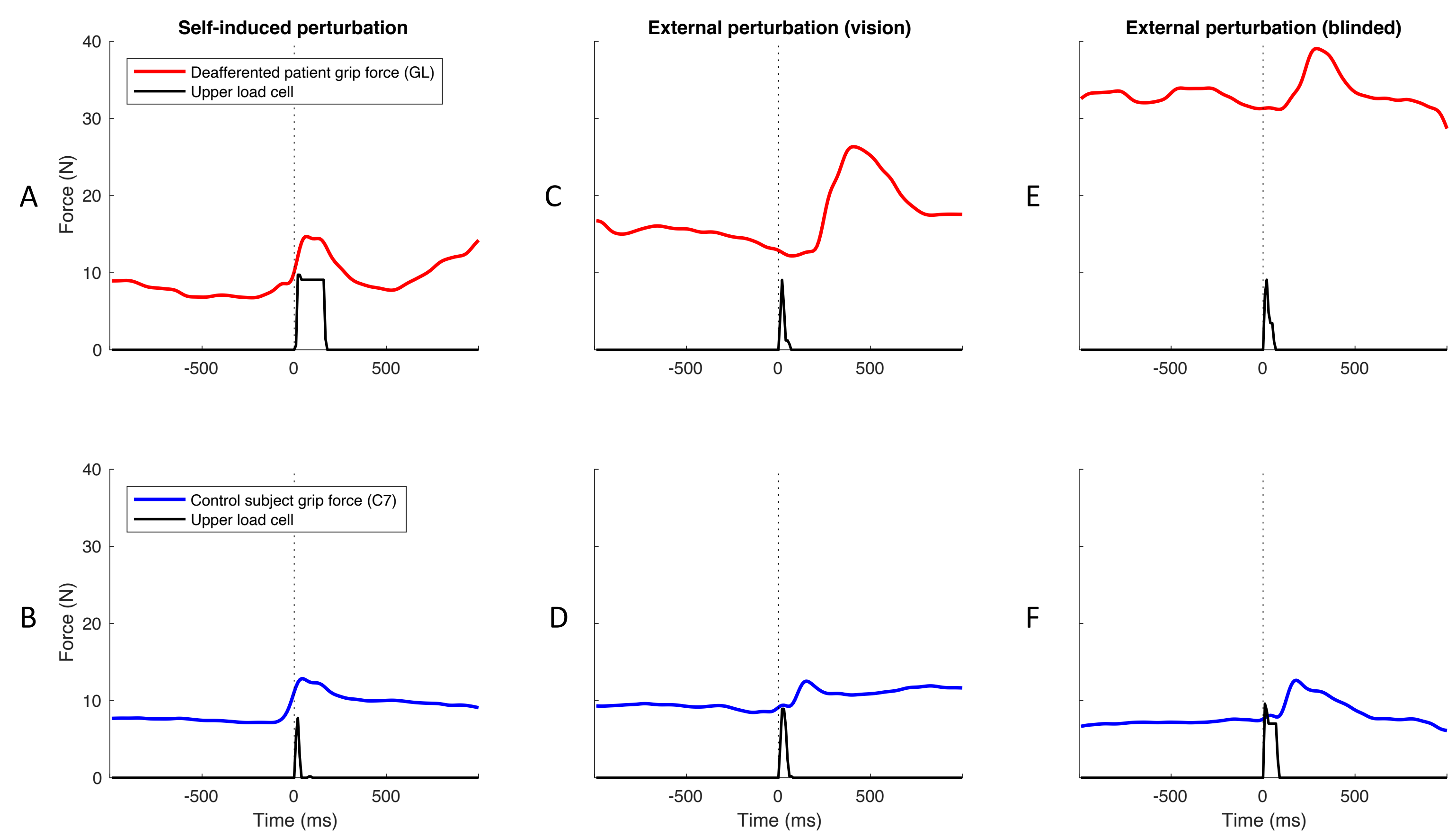

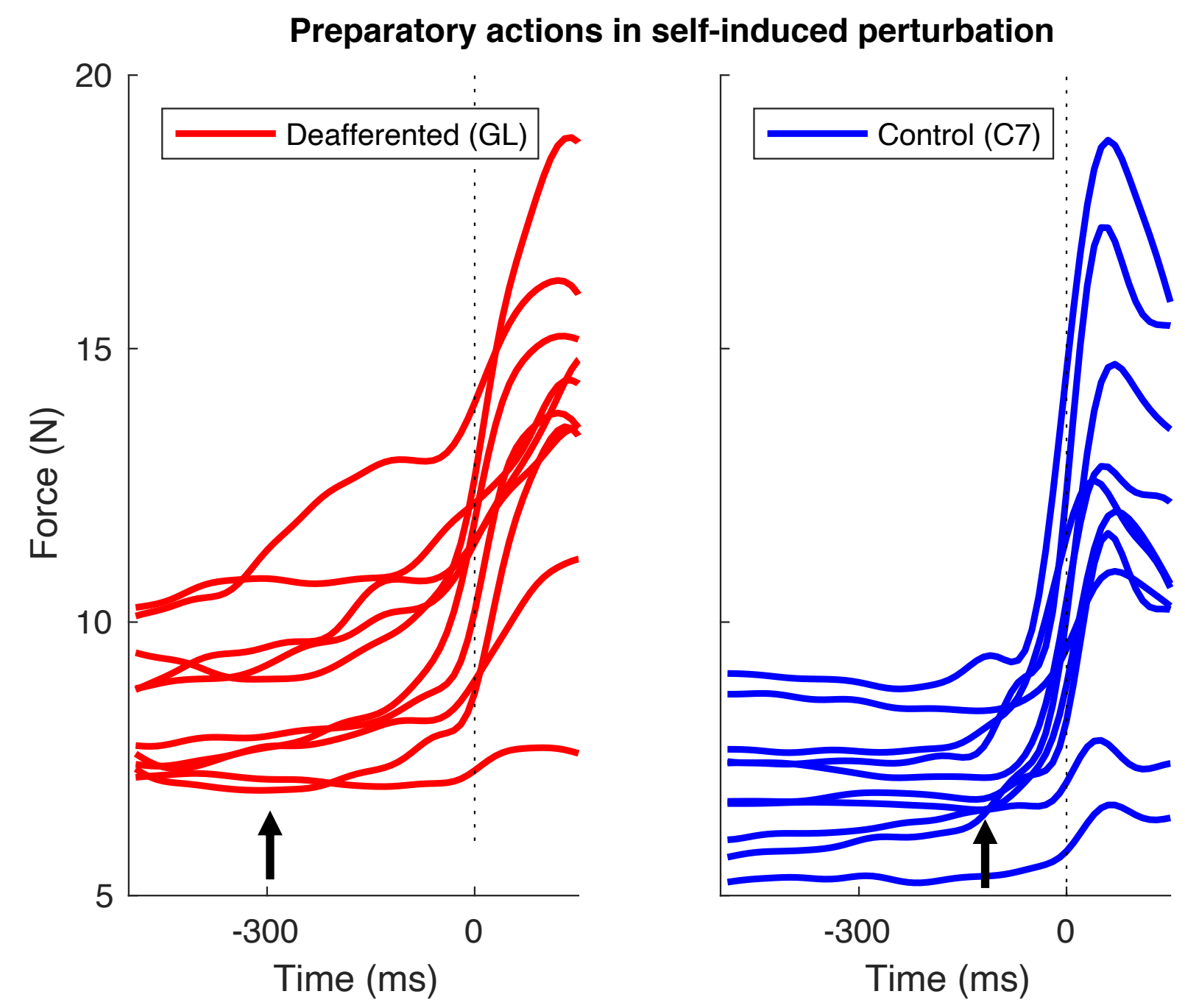

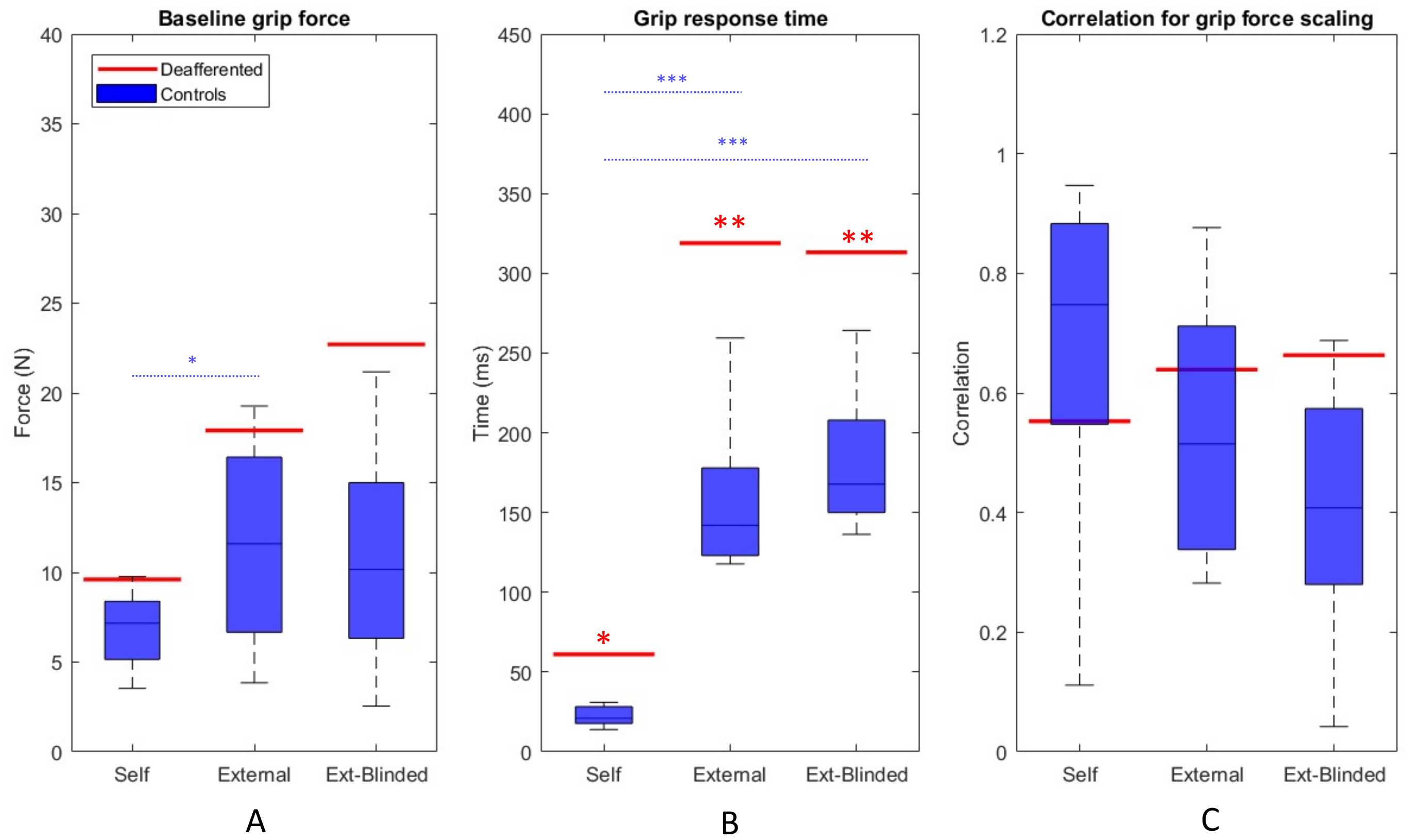

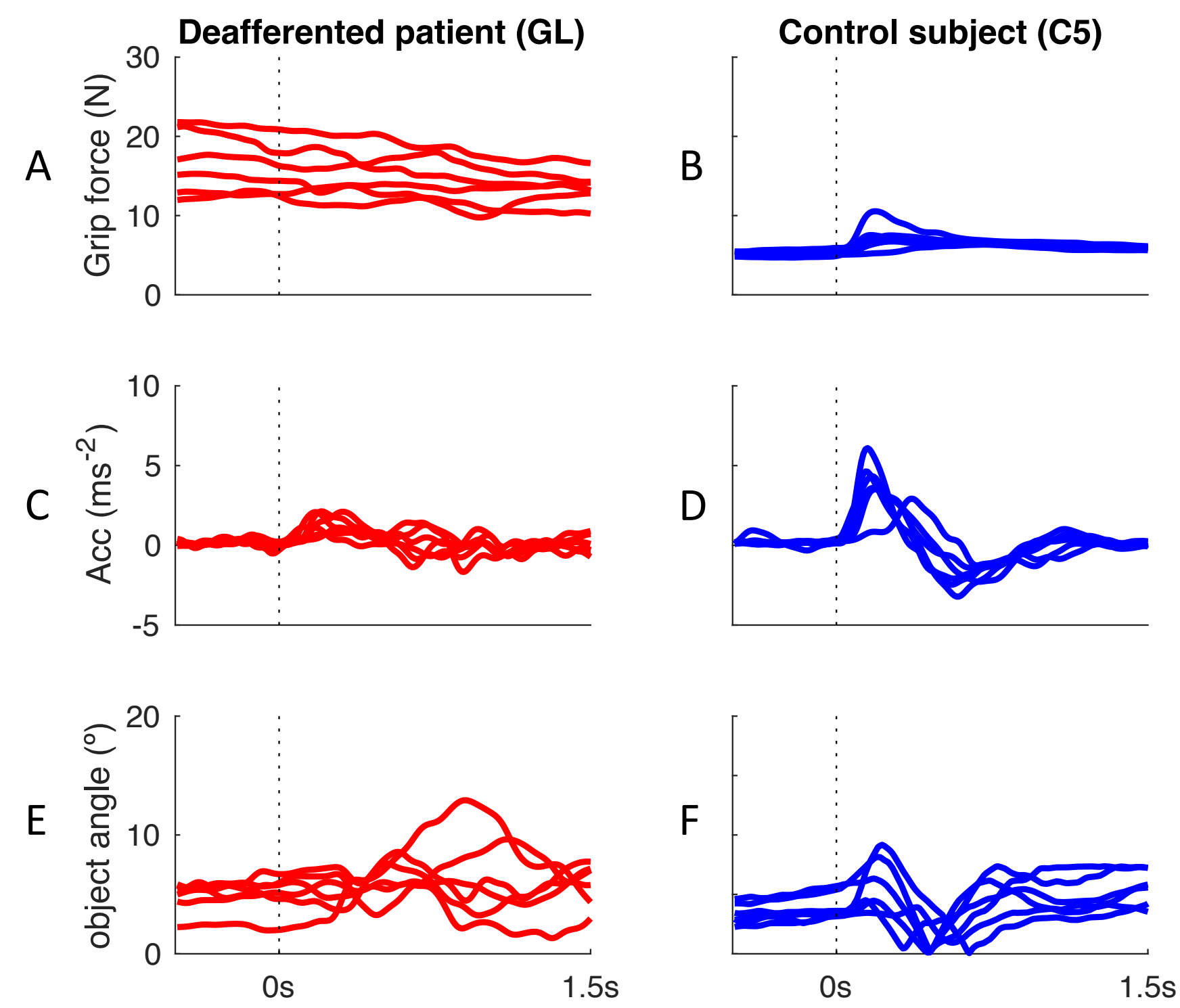

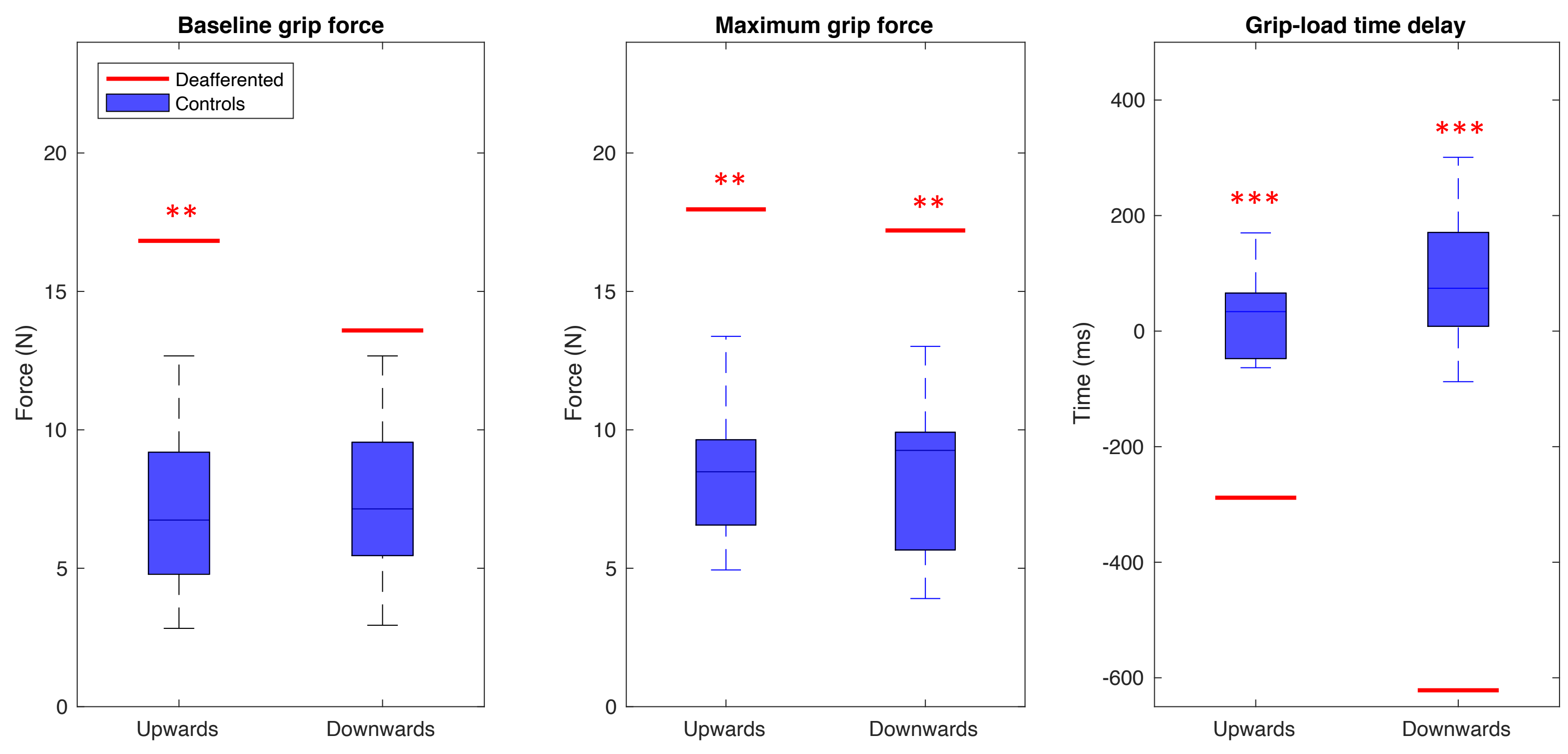

A 

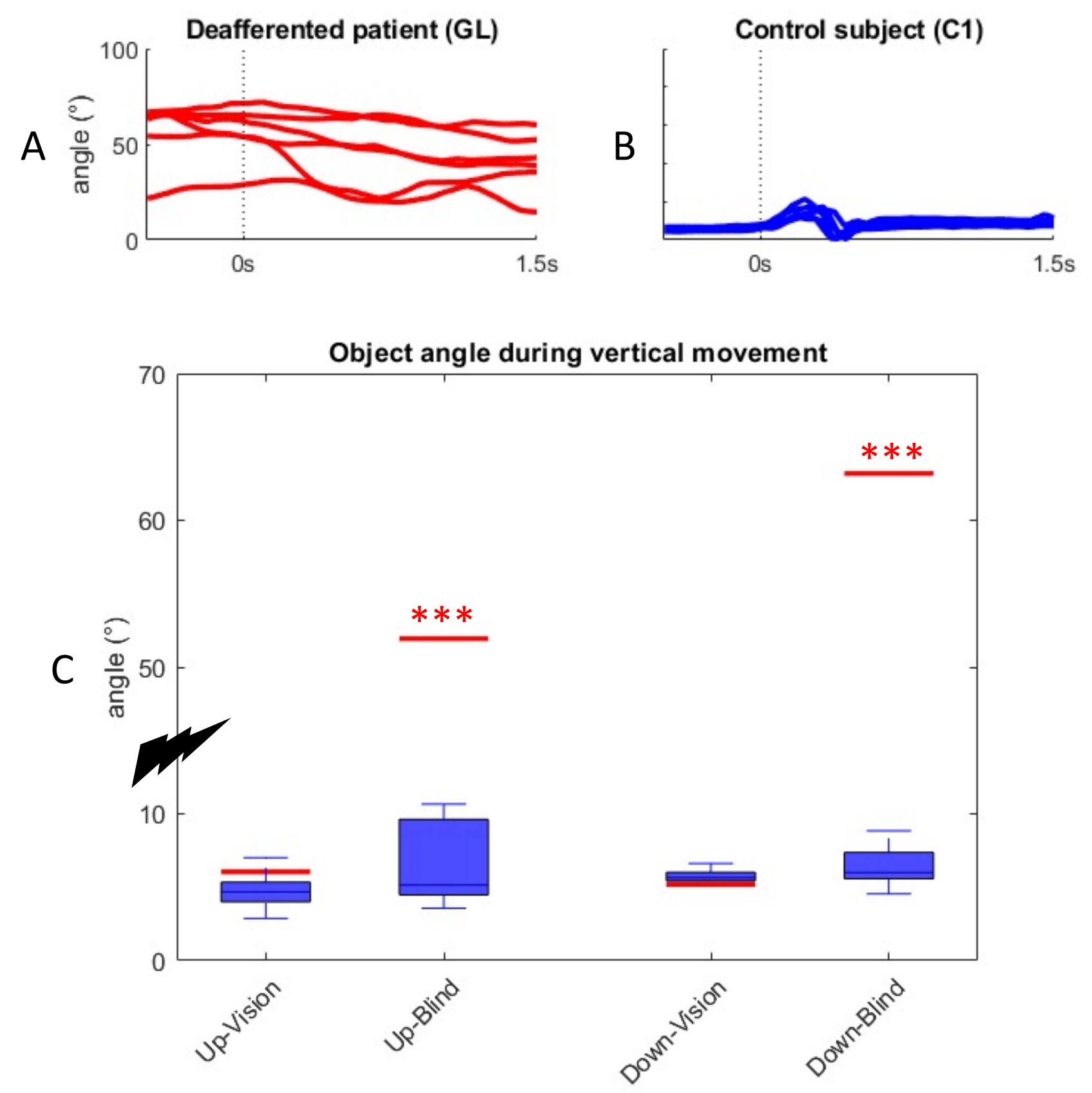

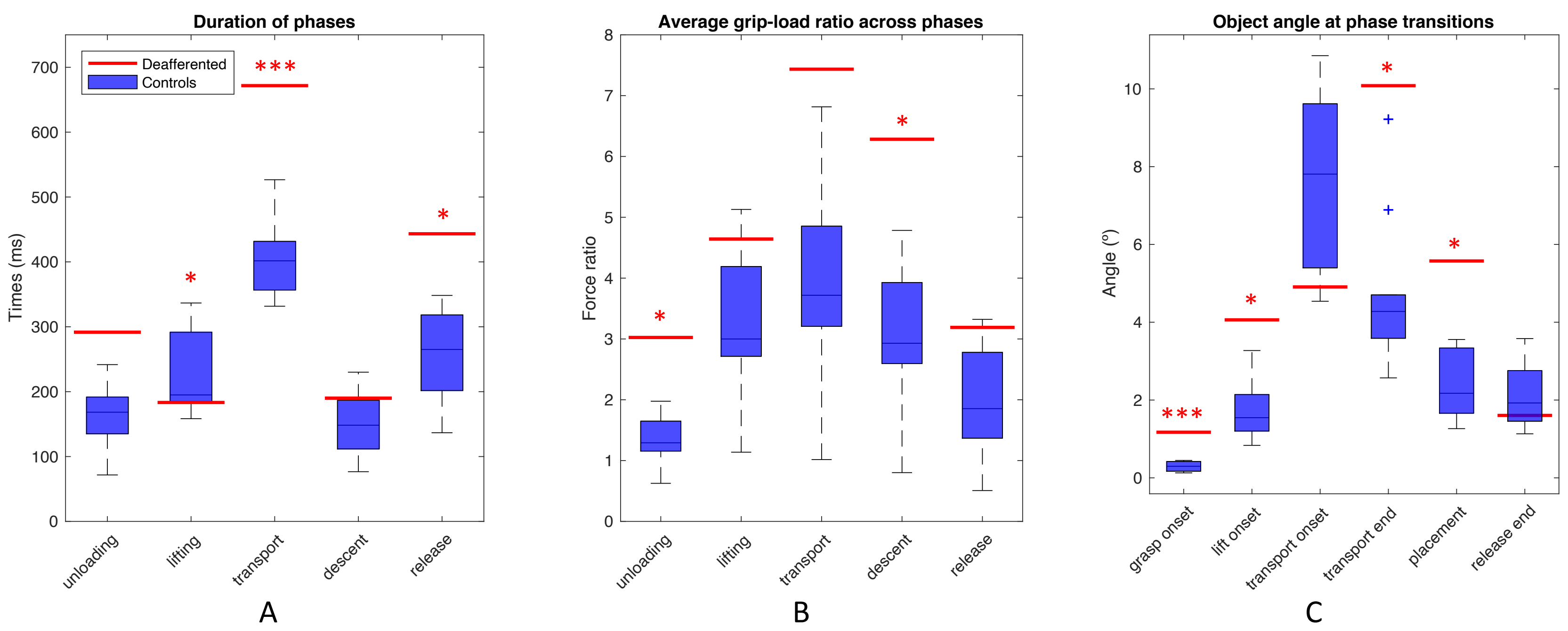
Touch and push errors upon task initiation

Deafferented

Controls

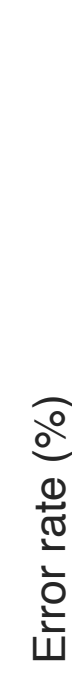

60

40

20

$0 \stackrel{+}{\text { Touch error prior to grasp onset }}$

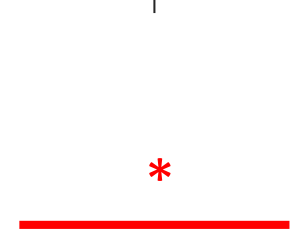

$*$

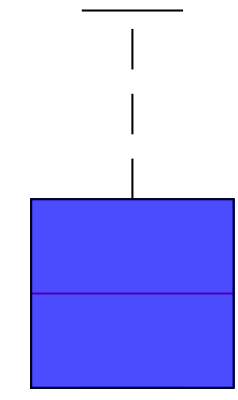

$+$

Push error during unloading

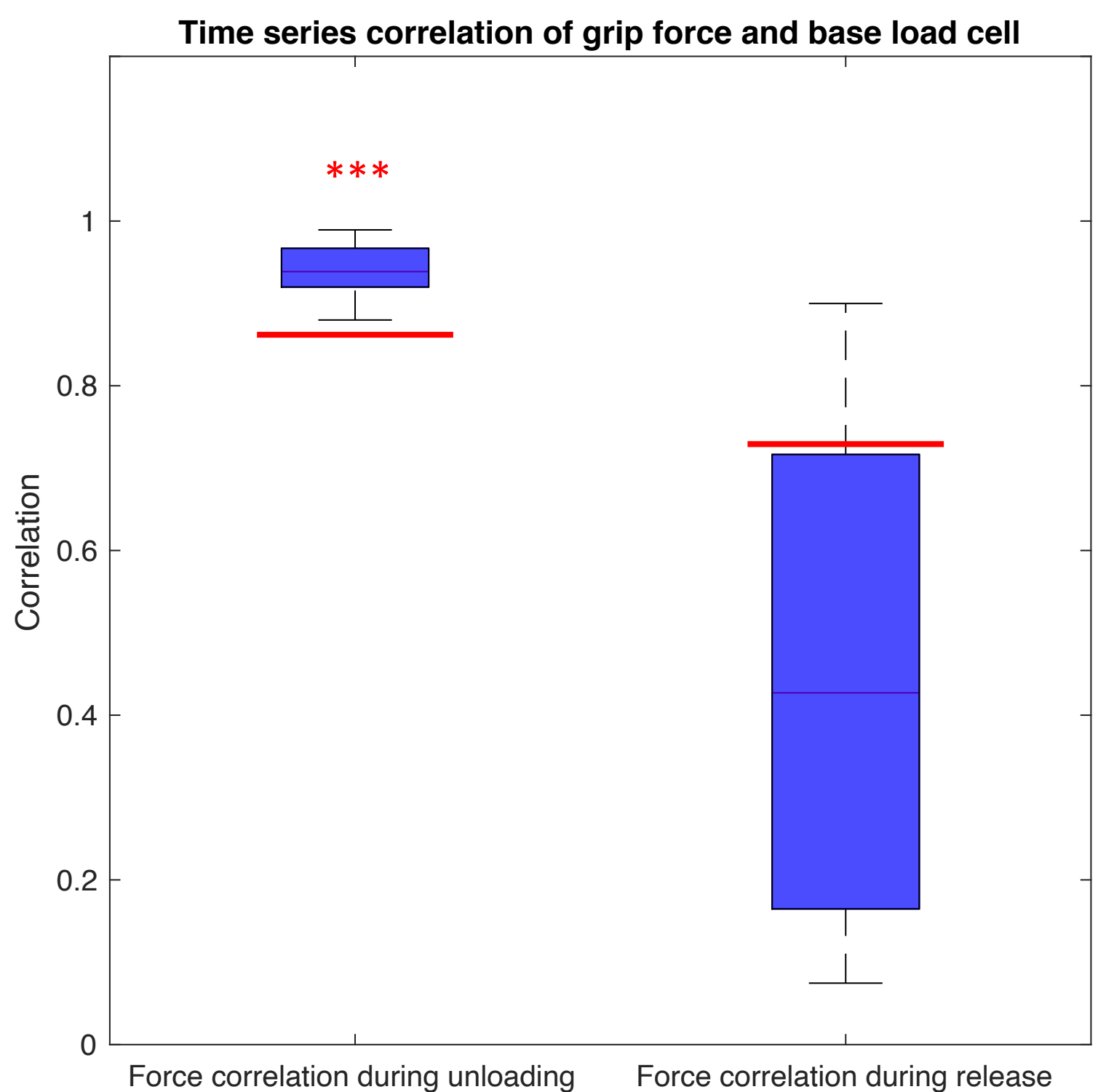




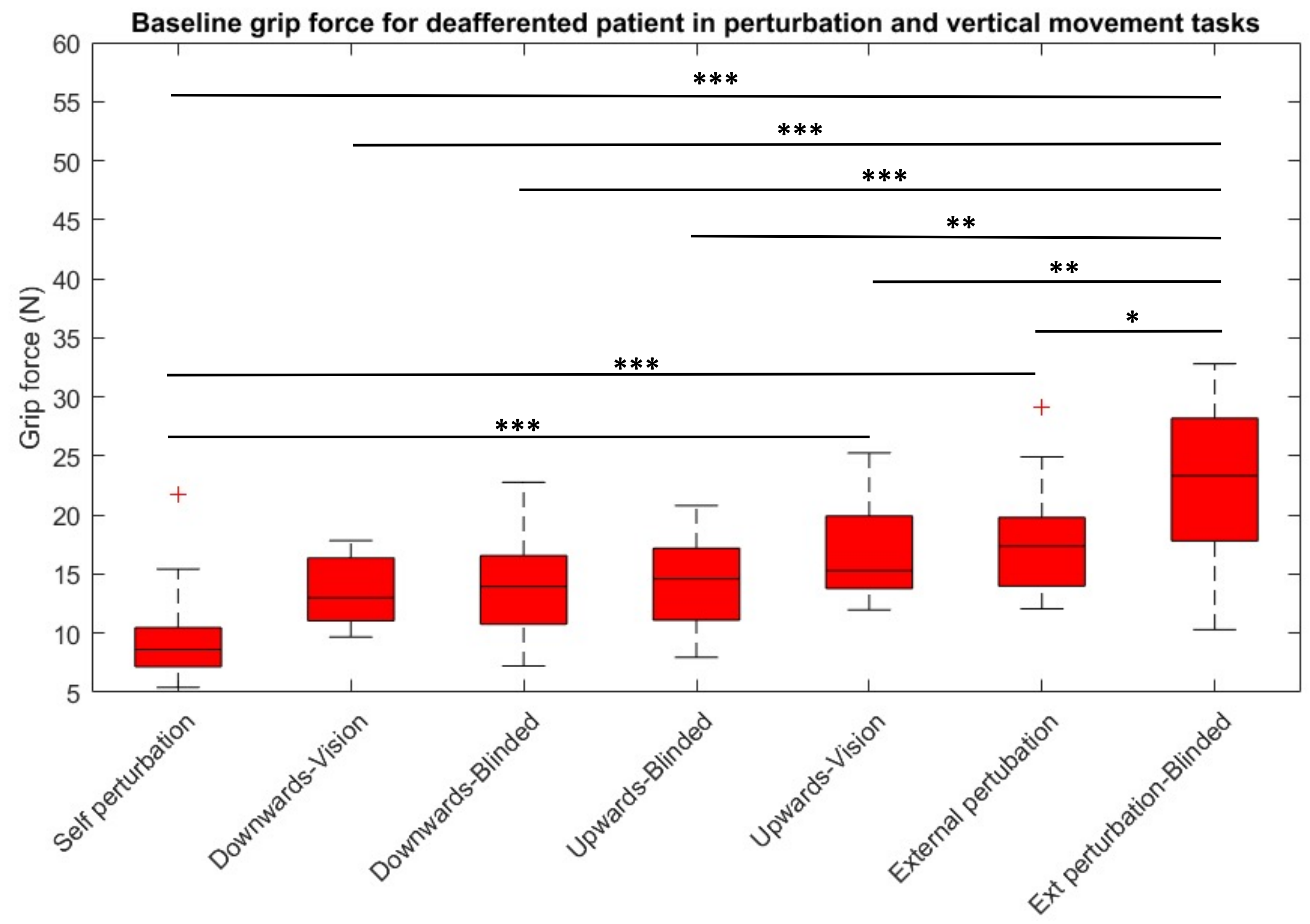




\section{Anticipation and compensation for somatosensory deficits in object handling: Evidence from a patient with large fibre sensory neuropathy}

Instrumented object with load cells, IMU

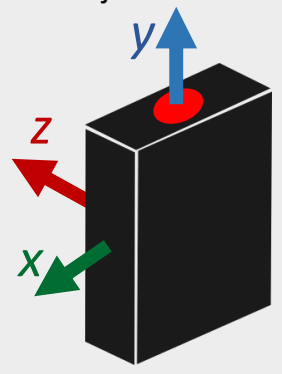

$\rightarrow$ grip force, acceleration, orientation
Object handling assessed in 1) perturbation to static holding; 2) vertical movement; 3) grasp and place tasks

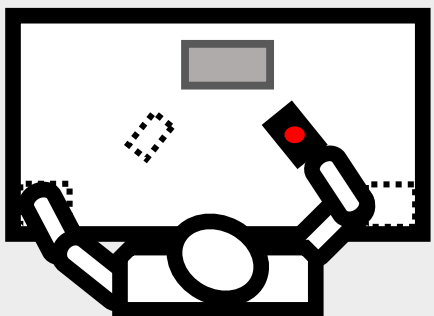

$\rightarrow$ comparison of deafferented patient and healthy controls
Increased grip forces on object

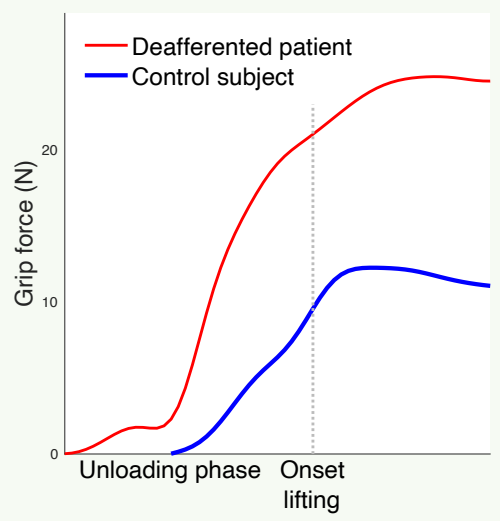

Changes in phase duration

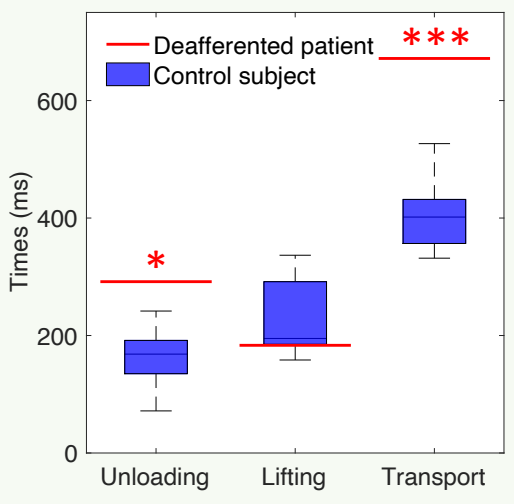

Object deviation at phase transitions

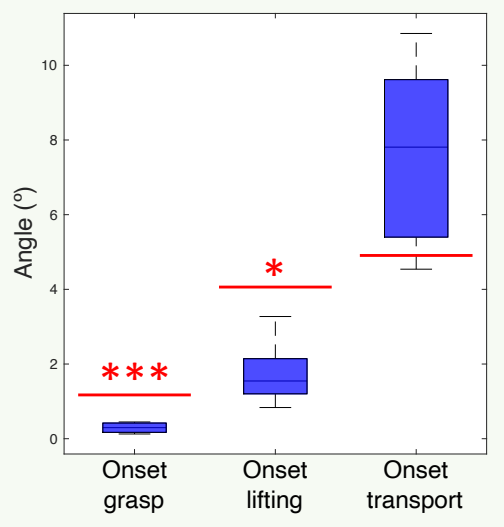

